\title{
Thermal ripples in bilayer graphene
}

\author{
Achille Mauri $\odot,{ }^{*}$ David Soriano $\odot$, and Mikhail I. Katsnelson $\odot$ \\ Radboud University, Institute for Molecules and Materials, Heyendaalseweg 135, 6525 AJ Nijmegen, The Netherlands
}

(Received 1 July 2020; revised 30 September 2020; accepted 1 October 2020; published 26 October 2020)

\begin{abstract}
We study thermal fluctuations of freestanding bilayer graphene subject to vanishing external tension. Within a phenomenological theory, the system is described as a stack of two continuum crystalline membranes, characterized by finite elastic moduli and a nonzero bending rigidity. A nonlinear rotationally invariant model guided by elasticity theory is developed to describe interlayer interactions. After neglection of in-plane phonon nonlinearities and anharmonic interactions involving interlayer shear and compression modes, an effective theory for soft flexural fluctuations of the bilayer is constructed. The resulting model, neglecting anisotropic interactions, has the same form of a well-known effective theory for out-of-plane fluctuations in a single-layer membrane, but with a strongly wave-vector-dependent bare bending rigidity. Focusing on AB-stacked bilayer graphene, parameters governing interlayer interactions in the theory are derived by first-principles calculations. Statistical-mechanical properties of interacting flexural fluctuations are then calculated by a numerical iterative solution of field-theory integral equations within the self-consistent screening approximation. The bare bending rigidity in the considered model exhibits a crossover between a long-wavelength regime governed by in-plane elastic stress and a short wavelength region controlled by monolayer curvature stiffness. Interactions between flexural fluctuations drive a further crossover between a harmonic and a strong-coupling regime, characterized by anomalous scale invariance. The overlap and interplay between these two crossover behaviors is analyzed at varying temperatures.
\end{abstract}

DOI: 10.1103/PhysRevB.102.165421

\section{INTRODUCTION}

The statistical properties of thermally fluctuating twodimensional (2D) membranes have been the subject of extensive investigations [1-3]. Crystalline layers, characterized by fixed connectivity between constituent atoms and a subsequent elastic resistance to compression and shear, exhibit a particularly rich thermodynamical behavior, both in clean and disordered realizations [1-16]. In the absence of substrates and without the action of an externally applied tension, fluctuations are only suppressed by elasticity and the bending rigidity of the layer. Although a naive application of the Mermin-Wagner theorem suggests the destruction of spontaneous order at any finite temperature, it has long been recognized that these freely fluctuating elastic membranes exhibit an orientationally ordered flat phase at low temperatures $[4,5]$. As a result of strong nonlinear coupling between bending and shear deformations, thermal fluctuations in the flat phase present anomalous scale invariance characterized by universal noninteger exponents. In the long-wavelength limit, the scale-dependent effective compression and shear moduli are driven to zero as power laws of the wave vector $q$, while the effective bending rigidity diverges as $\kappa(q) \approx$ $q^{-\eta}[6-8,10,11,17,18]$. This anomalous infrared behavior sets in at a characteristic Ginzburg scale $q_{*} \approx \sqrt{3 T Y /\left(16 \pi \kappa^{2}\right)}$, where $\kappa, Y$, and $T$ are, respectively, the bare bending rigidity, Young modulus, and temperature [3,19]. For shorter

\footnotetext{
*a.mauri@ science.ru.nl
}

wavelengths, $q>q_{*}$, within a membrane model based on continuum elasticity theory, fluctuation effects become negligible and the effective elastic moduli approach their bare values.

The first theoretical developments in the statistical mechanics of elastic membranes were driven by the physics of biological layers, polymerized membranes, and other surfaces $[1,2,20]$. After the isolation of atomically thin $2 \mathrm{D}$ materials, the relevance of statistical mechanical predictions for these extreme membrane realizations has raised vast interest, in both theory $[3,10,12,13,19,21,22]$ and experiments [23-27] (see also Refs. [28-32]).

In the case of atomically thin 2D membranes, numerical simulations with realistic atomic interactions are accessible [19,21,22,33-36], which allows material-specific predictions of the fluctuation behavior. Furthermore, the physics of graphene and other 2D materials stimulated new questions as compared to previously considered membrane realizations.

By exfoliation of graphite, it is possible to controllably extract multilayer membranes composed of $N$ stacked graphene sheets. As in the parent graphite structure, covalently bonded carbon layers are tied by weaker van der Waals interactions. The large difference between the strengths of covalent and interlayer binding forces generates an intriguing mechanical and statistical behavior, which has attracted vast research interest [37-42].

The properties of defect-free multilayers subject to small fluctuations, in the harmonic approximation, are already nontrivial. Mechanical properties are crucially determined by the coupling between interlayer shear deformation and out-of- 
plane, bending fluctuations. If layers are free to slide relative to each other at zero energy cost, we expect that the bending rigidity of the stack is controlled by the curvature stiffness of individual layers. We can thus assume that the bending rigidity is approximately $N \kappa$, where $N$ is the number of layers and $\kappa$ is the monolayer bare bending stiffness [39-42]. By contrast, the presence of a nonzero interlayer shear modulus forces layers to compress or dilate in response to curvature. Assuming rigid binding between layers, the bending stiffness is then controlled by in-plane elastic moduli and it grows proportionally to $N\left(N^{2}-1\right)$ for $N \geqslant 2$ [43]. For large $N$, the limiting $N^{3}$ scaling of the bending stiffness [39-43] is consistent with the continuum theory of thin elastic plates $[3,39,43,44]$. In the case of a graphene bilayer, the corresponding contribution to the bending rigidity can be written as $(B+\mu) l^{2} / 2$, where $B$ and $\mu$ are compression and shear moduli, and $l$ is the interlayer distance [39].

A theory interpolating between these extreme regimes was developed, within a harmonic approximation, in Ref. [39]. As a modeling framework, the system was described as a stack of continuum 2D elastic media. The energy functional describing coupling between layers was constructed by discretizing the continuum theory of a 3D uniaxial solid. Within this model, coupled and decoupled fluctuation regimes are recovered as limiting cases for long and short wavelengths, connected by a crossover: Coupling between flexural and interlayer shear deformations sets in for wave vectors smaller than characteristic scales determined by elastic stiffnesses and interlayer interactions [39].

Recent experimental measurements of the bending rigidity $[37,40,42,45,46]$ present a large scatter and indicate smaller values compared to the theoretical prediction for the long-wavelength, rigidly coupled case. In the case of bilayer graphene, different experimental techniques lead to

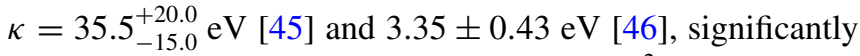
smaller than the elastic contribution $(B+\mu) l^{2} / 2$, which corresponds to a rigidity of the order of $100 \mathrm{eV}$ (theoretical predictions in Ref. [43] lead to $\kappa \simeq 160 \mathrm{eV}$ ). For few-layer membranes with $N \geqslant 2$, Ref. [45] reported evidence that the overall bending rigidity scales as $N^{2}$. More recently, by analyzing pressurized bubbles in multilayer graphene, $\mathrm{MoS}_{2}$, and hexhagonal BN, Ref. [40] reported values of $\kappa$ intermediate between the uncoupled limit $N \kappa$ and the rigidlycoupled case, and interpreted the observed behavior as the result of interlayer slippage between atomic planes. Finally, Ref. [42] observed multilayer graphene membranes under varying bending angles. Values of the bending stiffness close to $N \kappa$ were observed for large angles, which was interpreted by a dislocation model of interlayer slippage.

Reported results for the interlayer shear modulus in multilayer graphene also exhibit a large dispersion, see, e.g., Ref. [37].

At finite temperatures, statistical properties of fluctuating stacks of crystalline membranes have been long investigated. A rich physics was predicted in early studies motivated by lamellar phases of polymerized membranes. In particular, Ref. [47] predicted a sharp phase transition between a coupled state and a decoupled phase, in which algebraic decay of crystalline translational order makes interlayer shear coupling irrelevant. Refs. [48,49] elaborated on the properties of the decoupled state, within a nonlinear 3D continuum theory and determined logarithmic renormalizations due to thermal fluctuations.

In the context of crystalline bilayer and multilayer graphene membranes, finite-temperature anharmonic lattice fluctuations were extensively addressed by numerical simulations (see, e.g., Refs. [34,36,50]).

In this paper, we study thermal fluctuations of ideal, defect-free bilayer graphene within a phenomenological, elasticitylike model. The theory of Ref. [39] is assumed as a starting point and generalized to include crucial nonlinearities which control anomalous scaling behavior. An interesting aspect introduced by finite temperatures stems from the interplay of different wave vector scales: characteristic scales marking the onset of coupling between flexural and interlayer shear, and Ginzburg scales $q_{*}$ controlling the transition from harmonic to strongly nonlinear fluctuations. To obtain a global picture of correlation functions at arbitrary wave vector $q$, we derive a numerical solution of Dyson equations within the self-consistent screening approximation (SCSA) $[9,10,51]$. In the long wavelength limit, the universal power-law behavior predicted by membrane theory is recovered and the SCSA scaling exponent $\eta=4 /(1+\sqrt{15})$ is reproduced with high accuracy. The finite-wavelength solution, furthermore, gives access to crossovers in correlation functions and to nonuniversal properties specific to bilayer graphene. To develop material-specific predictions, we develop an ab initio prediction of model parameters focusing on the case of AB-stacked bilayer graphene.

The paper is organized as follows: In Sec. II, after a brief discussion of theories for single-layer membranes, we introduce a phenomenological model which extends the theory of Ref. [39] with the inclusion of nonlinearities required by rotational invariance. Subsequently, the model is simplified by neglecting all nonlinearities but interactions of the collective out-of-plane displacement field. In Sec. IIC, we derive an effective model for flexural fluctuations by successively integrating out all other fields. After neglecting anisotropic interactions, this model takes the form of a standard theory for crystalline membranes, with a strongly $q$-dependent bare bending rigidity. In Sec. III, we discuss model parameters for AB-stacked bilayer graphene and describe first-principles calculations of the interlayer coupling moduli. In Sec. IV, correlation functions of the resulting model are calculated at an arbitrary wave vector within the SCSA [9,10,51]; an iterative algorithm is used to determine numerical solutions of SCSA equations. Results are illustrated in Sec. V. Finally, Sec. VI discusses an extension to the theory in which nonlinearities in flexural fields of both layers are taken into account. Section VII summarizes and concludes the paper.

\section{MODEL}

\section{A. Single layer}

This section briefly introduces existing models for 2D crystalline membranes, extensively discussed in Refs. [1-8,10,12].

In a long-wavelength continuum limit, membrane configurations are specified by the coordinates $\mathbf{r}(\mathbf{x})$ in $3 \mathrm{D}$ space of mass points in the $2 \mathrm{D}$ crystal, identified by an internal 
2D coordinate $\mathbf{x}$. After specification of an energy functional $H[\mathbf{r}(\mathbf{x})]$, the statistics of fluctuating configurations at a temperature $T$ is governed by the Gibbs probability distribution,

$$
P[\mathbf{r}(\mathbf{x})]=\frac{1}{Z} \mathrm{e}^{-H_{0}[\mathbf{r}(\mathbf{x})] / T},
$$

where

$$
Z=\int[\mathrm{d} \mathbf{r}(\mathbf{x})] \mathrm{e}^{-H_{0}[\mathbf{r}(\mathbf{x})] / T}
$$

is the partition function, and $\int[\mathrm{d} \mathbf{r}(\mathbf{x})]$ denotes functional integration over the field $\mathbf{r}(\mathbf{x})$.

In the spirit of elasticity theory, a model for membranes with nonzero stiffness to curvature and strain is defined by the configuration energy $[5,7,8]$,

$$
H_{0}=\int \mathrm{d}^{2} x\left[\frac{\kappa}{2}\left(\partial^{2} \mathbf{r}\right)^{2}+\frac{\lambda}{2}\left(U_{\alpha \alpha}\right)^{2}+\mu U_{\alpha \beta} U_{\alpha \beta}\right],
$$

where $\kappa, \lambda$, and $\mu$ are, respectively, the bending rigidity and Lamé elastic coefficients. The notation $\partial_{\alpha}=\partial / \partial x_{\alpha}$ indicates differentiation with respect to internal coordinates, and $U_{\alpha \beta}=$ $\frac{1}{2}\left(\partial_{\alpha} \mathbf{r} \cdot \partial_{\beta} \mathbf{r}-\delta_{\alpha \beta}\right)$ is the strain tensor, proportional to the local deformation of the metric $g_{\alpha \beta}=\partial_{\alpha} \mathbf{r} \cdot \partial_{\beta} \mathbf{r}$ from the Euclidean metric $\delta_{\alpha \beta}$. In Eq. (3), mass points are labeled via their coordinates in a configuration of mechanical equilibrium: Reference coordinates $x_{1}, x_{2}$ are chosen in such way that states of minimum energy are $\mathbf{r}(\mathbf{x})=x_{\alpha} \mathbf{v}_{\alpha}+\mathbf{t}=x_{1} \mathbf{v}_{1}+x_{2} \mathbf{v}_{2}+\mathbf{t}$, where $\mathbf{v}_{1}, \mathbf{v}_{2}$ is any given pair of mutually orthogonal unit vectors and $\mathbf{t}$ is an arbitrary constant vector.

The energy functional Eq. (3) has been extensively discussed as a Landau-Ginzburg model for critical phenomena at the crumpling transition and also as a starting point to discuss scaling properties of the flat phase $[1,2,5,8,11]$.

In the flat phase, it is convenient to parametrize $\mathbf{r}(\mathbf{x})=$ $\left(x_{\alpha}+u_{\alpha}(\mathbf{x})\right) \mathbf{v}_{\alpha}+h(\mathbf{x}) \mathbf{n}$, where $\mathbf{n}=\mathbf{v}_{1} \times \mathbf{v}_{2}$ denotes the normal to the membrane plane. Assuming that displacement fields and their gradients are small, such that $\left|\partial^{2} u\right| \ll|\partial u|$ and $|\partial u| \ll 1$, Eq. (3) can be reduced by the replacements $U_{\alpha \beta} \rightarrow u_{\alpha \beta}=\left(\partial_{\alpha} u_{\beta}+\partial_{\beta} u_{\alpha}+\partial_{\alpha} h \partial_{\beta} h\right) / 2,\left(\partial^{2} \mathbf{r}\right)^{2} \rightarrow\left(\partial^{2} h\right)^{2}$, which leads to the standard approximate form $[1,4,6,12,52]$

$$
\tilde{H}_{0}=\int \mathrm{d}^{2} x\left[\frac{\kappa}{2}\left(\partial^{2} h\right)^{2}+\frac{\lambda}{2} u_{\alpha \alpha}^{2}+\mu u_{\alpha \beta}^{2}\right] .
$$

The neglected terms are expected to be unnecessary for an exact calculation of universal quantities such as scaling exponents. This is supported by a power-counting argument in the framework of a field-theoretic $\varepsilon$-expansion method [6]: After extension of the problem to $D$-dimensional membranes in a $d$-dimensional embedding space, neglected terms are irrelevant by power counting at the upper critical dimension $D=4$. Equation (4) thus plays the role of an effective theory $[6,8]$ suitable for calculation of scaling indices to all orders in an $\varepsilon=(4-D)$ expansion.

In the transition from Eq. (3) to Eq. (4), neglected nonlinearities lead to an explicit breaking of rotational symmetry. However, as is well known $[2,8]$, the underlying invariance is preserved in a deformed form: $\tilde{H}_{0}$ is invariant under the transformations

$$
\begin{aligned}
h(\mathbf{x}) & \rightarrow h(\mathbf{x})+A_{\alpha} x_{\alpha}+B, \\
u_{\alpha}(\mathbf{x}) & \rightarrow u_{\alpha}(\mathbf{x})-A_{\alpha} h(\mathbf{x})-\frac{1}{2} A_{\alpha} A_{\beta} x_{\beta}+B_{\alpha}^{\prime}
\end{aligned}
$$

for arbitrary coordinate-independent $A_{\alpha}, B$, and $B_{\alpha}^{\prime}$. This deformed symmetry and the subsequent Ward identities are crucial in the renormalization of the theory of membranes, and, most importantly, in the protection of the softness of flexural modes, which ensures the criticality of the theory without fine tuning of parameters [2,6,8-10].

It is useful to compare Eqs. (3) and (4) with the CanhamHelfrich model for fluid membranes [1] and with the model for crystalline membranes developed in Ref. [6]. In Ref. [6], bending rigidity of the layer was introduced via an energy contribution of the form

$$
\frac{\kappa}{2} \int \mathrm{d}^{2} x\left(\partial_{\alpha} \mathbf{N}\right)^{2}=\frac{\kappa}{2} \int \mathrm{d}^{2} x \mathbf{K}_{\beta}^{\alpha} \cdot \mathbf{K}_{\alpha \beta},
$$

where $\mathbf{N}$ is the local normal to the surface, $\mathbf{K}_{\alpha \beta}$ is the curvature tensor, and $\mathbf{K}_{\beta}^{\alpha}=g^{\alpha \gamma} \mathbf{K}_{\beta \gamma}$. Using that $\mathbf{K}_{\alpha \beta}=\mathbf{N}\left(\mathbf{N} \cdot \partial_{\alpha} \partial_{\beta} \mathbf{r}\right)$ for 2D surfaces (see, e.g., Chap. 7 of Ref. [1]), Eq. (6) can be written as

$$
\frac{\kappa}{2} \int \mathrm{d}^{2} x g^{\beta \gamma}\left(\mathbf{N} \cdot \partial_{\alpha} \partial_{\beta} \mathbf{r}\right)\left(\mathbf{N} \cdot \partial_{\alpha} \partial_{\gamma} \mathbf{r}\right) .
$$

Here $g^{\alpha \beta}$ denotes the inverse matrix of the metric tensor $g_{\alpha \beta}=$ $\partial_{\alpha} \mathbf{r} \cdot \partial_{\beta} \mathbf{r}$. For small fluctuations, such that $g_{\alpha \beta} \simeq \delta_{\alpha \beta}$ and $\mathbf{N} \simeq$ $\mathbf{n}$, the curvature energy reduces, at leading order, to

$$
\begin{aligned}
& \frac{\kappa}{2} \int \mathrm{d}^{2} x \partial_{\alpha} \partial_{\beta} h \partial_{\alpha} \partial_{\beta} h \\
& \quad=\frac{\kappa}{2} \int \mathrm{d}^{2} x\left[\left(\partial^{2} h\right)^{2}+\left(\delta_{\alpha \beta} \partial^{2}-\partial_{\alpha} \partial_{\beta}\right)\left(\partial_{\alpha} h \partial_{\beta} h\right)\right],
\end{aligned}
$$

which, up to boundary terms, is equivalent to

$$
\frac{\kappa}{2} \int \mathrm{d}^{2} x\left(\partial^{2} h\right)^{2}
$$

the curvature term in Eq. (4).

In the Canham-Helfrich model [1], the curvature stiffness for a fluid membrane with vanishing spontaneous curvature reads

$$
\int \mathrm{d}^{2} x \sqrt{g}\left[2 \kappa_{b} H^{2}+\kappa_{G} K\right]
$$

where $H$ and $K$ are the mean and the Gaussian curvature, and $g=\operatorname{det}\left[g_{\alpha \beta}\right]$.

For 2D surfaces [1],

$$
H=\frac{1}{2} K_{\alpha}^{\alpha} \quad \text { and } \quad K=\frac{1}{2}\left[\left(K_{\alpha}^{\alpha}\right)^{2}-K_{\beta}^{\alpha} K_{\alpha}^{\beta}\right],
$$

where $K_{\alpha \beta}=\mathbf{N} \cdot \partial_{\alpha} \partial_{\beta} \mathbf{r}, K_{\beta}^{\alpha}=g^{\alpha \gamma} K_{\beta \gamma}$. The Canham-Helfrich energy functional Eq. (10) is reparametrization invariant, expressing that the configuration energy is only sensitive to the geometrical shape of the surface in 3D space and not on its internal coordinate system. As was discussed in Ref. [6], in crystalline layers the crystal lattice singles out a natural parametrization of the membrane and reparametrization invariance is not a necessary requirement (see also Ref. [8] for a more general discussion in presence of nonflat internal metric). 
For small fluctuations about a flat configuration, the mean and Gaussian curvatures reduce to

$$
H \simeq \frac{1}{2}\left(\partial^{2} h\right)
$$

and

$$
\begin{aligned}
K & \simeq \frac{1}{2}\left[\left(\partial^{2} h\right)^{2}-\left(\partial_{\alpha} \partial_{\beta} h\right)\left(\partial_{\alpha} \partial_{\beta} h\right)\right] \\
& =-\frac{1}{2}\left(\delta_{\alpha \beta} \partial^{2}-\partial_{\alpha} \partial_{\beta}\right)\left(\partial_{\alpha} h \partial_{\beta} h\right),
\end{aligned}
$$

while $\sqrt{g} \simeq 1$. Integration over $K$ then leads to a boundary term and a curvature energy density proportional to $\left(\partial^{2} h\right)^{2}$ is recovered. More generally, the Gauss-Bonnet theorem implies that the integral $\int \mathrm{d}^{2} x \sqrt{g} K$ is topological invariant for closed surfaces and the sum of boundary terms and a topological invariant for open surfaces.

In this paper, curvature energy is considered to a leading order in the limit of small fluctuations about a flat configuration, and boundary terms arising from the surface integration of the leading-order Gaussian curvature, Eq. (13), are neglected.

We note, however, that the Gaussian curvature energy plays an important role in processes which involve a change of membrane topology [1] or finite-size membranes with a boundary. For example, a recent analysis of thermal fluctuations within the harmonic approximation [53] indicated an important role of Gaussian curvature energy in the statistics of fluctuating membranes with a free edge. Finally, we note that models with higher-order powers of curvature were considered in Refs. [54,55], in relation with the problem of bolaamphiphilic vesicles.

As a concluding remark, we notice that the models discussed above assume locality of the configuration energy and, therefore, do not include infinite-range forces such as van der Waals [56], dipole interactions [57], or the coupling with gapless electrons, discussed in connection with graphene in Refs. [58,59].

\section{B. Bilayer}

We will model bilayer graphene as a stack of two coupled elastic membranes [39]. The corresponding energy functional can thus be written as

$$
H=\sum_{i=1}^{2} H_{i}+H_{c},
$$

where

$$
H_{i}=\int \mathrm{d}^{2} x\left[\frac{\kappa}{2}\left(\partial^{2} \mathbf{r}_{i}\right)^{2}+\frac{\lambda}{2} U_{i \alpha \alpha}^{2}+\mu U_{i \alpha \beta}^{2}\right]
$$

are single-layer energies, and $H_{c}$ represents coupling between membranes [60]. In Eq. (15), $\mathbf{r}_{i}$ and $U_{i \alpha \beta}$ denote the coordinates and the local deformation tensor of the $i$ th layer in the stack. As a model for interlayer interactions, we assume a local coupling [61] truncated at the leading order in a gradient expansion. This corresponds to an energy functional of the form

$$
H_{c}=\int \mathrm{d}^{2} x \mathcal{H}_{c}(\mathbf{x})
$$

with an energy density $\mathcal{H}_{c}(\mathbf{x})$ depending only on $\mathbf{r}_{1}(\mathbf{x})$ and $\mathbf{r}_{2}(\mathbf{x})$ and their leading-order gradients at $\mathbf{x}$. After introduction (a)

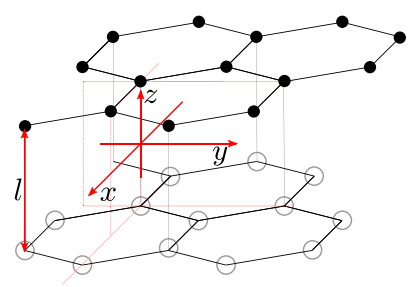

(b)

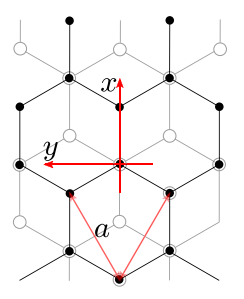

FIG. 1. (a) Lattice structure of bilayer graphene in the Bernal (AB) stacking. (b) Top view of AB-stacked bilayer graphene.

of sum and difference coordinates $\mathbf{r}=\frac{1}{2}\left(\mathbf{r}_{1}+\mathbf{r}_{2}\right), \mathbf{s}=\mathbf{r}_{1}-$ $\mathbf{r}_{2}$, invariance under translations in the $3 \mathrm{D}$ ambient space implies that $\mathcal{H}_{c}$ cannot depend on $\mathbf{r}$ but only on its derivatives. In the leading order of a gradient expansion, we will thus assume that $\mathcal{H}_{c}(\mathbf{x})$ depends only on the local separation vector $\mathbf{s}$ and on the tangent vectors $\partial_{\alpha} \mathbf{r}$, neglecting dependence on higher derivatives such as $\partial_{\alpha} \mathbf{s}$ or $\partial^{2} \mathbf{r}$. This level of approximation is analogous to the approach in Ref. [39], where the coupling energy is derived by discretization of a continuum 3D elasticity theory. In the following, we will assume the developed elasticitylike theory as a model to describe finite-wavelength phenomena.

The most general form of $\mathcal{H}_{c}$ depending on $\mathbf{s}$ and $\partial_{\alpha} \mathbf{r}$ and consistent with rotational and inversion symmetries of the 3D ambient space is a generic function of the scalar products [62]

$$
\partial_{\alpha} \mathbf{r} \cdot \partial_{\beta} \mathbf{r}, \quad \mathbf{s} \cdot \partial_{\alpha} \mathbf{r}, \quad s^{2} .
$$

In the configuration of mechanical equilibrium, neglecting a small uniform strain induced by interlayer coupling, $\mathbf{r}(\mathbf{x})=$ $x_{\alpha} \mathbf{v}_{\alpha}$ and the relative displacement between layers is $\mathbf{s}(\mathbf{x})=$ $l \mathbf{n}$, where $l$ is the interlayer distance and $\mathbf{n}=\mathbf{v}_{1} \times \mathbf{v}_{2}$. For small fluctuations, the coupling energy can thus be expanded in powers of the strain tensor $U_{\alpha \beta}=\frac{1}{2}\left(\partial_{\alpha} \mathbf{r} \cdot \partial_{\beta} \mathbf{r}-\delta_{\alpha \beta}\right)$, the field $\mathbf{s} \cdot \partial_{\alpha} \mathbf{r}$, which measures interlayer shear, and $s^{2}-l^{2}$, which describes local dilations of the layer-to-layer distance.

Consistency with the dihedral $D_{3 d}$ symmetry of $\mathrm{AB}$ stacked bilayer graphene [63] [see Figs. 1(a) and 1(b)] selects, among general combinations of these terms, a subset of allowed invariant functions. Symmetry-consistent terms can be directly constructed by group theory arguments or, equivalently, by adapting invariants from theory of 3D elastic media. Identification of $\mathbf{s} / l=\left(\mathbf{r}_{1}-\mathbf{r}_{2}\right) / l$ with a discrete version of $\partial_{z} \mathbf{r}$ in a corresponding $3 \mathrm{D}$ theory indicates that $\mathbf{s} \cdot \partial_{\alpha} \mathbf{r} / l$ and $\left(s^{2}-l^{2}\right) / l^{2}$ have the same transformation properties of strain tensor components $U_{\alpha z}=\partial_{\alpha} \mathbf{r} \cdot \partial_{z} \mathbf{r}$ and $U_{z z}=\partial_{z} \mathbf{r} \cdot \partial_{z} \mathbf{r}-1$, respectively. The general elastic free-energy of uniaxial media with $D_{3 d}$ point group subject to uniform deformation reads $[44,64]$

$$
\begin{aligned}
F= & \frac{1}{2} \bar{C}_{1}\left(U_{\alpha \alpha}\right)^{2}+\bar{C}_{2} U_{\alpha \beta} U_{\alpha \beta} \\
& +\frac{1}{2} C_{1} U_{z z}^{2}+\frac{1}{2} C_{2} U_{\alpha z}^{2}+C_{3} U_{\alpha \alpha} U_{z z} \\
& +C_{4}\left[\left(U_{x x}-U_{y y}\right) U_{x z}-2 U_{x y} U_{y z}\right],
\end{aligned}
$$

where Greek indices run over $x$ and $y$ components and $\bar{C}_{i}$ and $C_{i}$ are constants. In Eq. (18) and in the following, referencespace coordinates are interchangeably denoted as $\left(x_{1}, x_{2}\right)$ or 
$(x, y)$. Returning to the bilayer case, by drawing from analogous invariants in Eq. (18), we can write the functional $H_{c}$ as

$$
\begin{aligned}
H_{c}= & \int \mathrm{d}^{2} x\left[\frac{g_{1}}{8 l^{4}}\left(s^{2}-l^{2}\right)^{2}+\frac{g_{2}}{2 l^{2}}\left(\mathbf{s} \cdot \partial_{\alpha} \mathbf{r}\right)^{2}\right. \\
& +\frac{g_{3}}{4 l^{2}}\left(s^{2}-l^{2}\right) U_{\alpha \alpha}+\frac{g_{4}}{2 l}\left(\left(\mathbf{s} \cdot \partial_{x} \mathbf{r}\right)\left(U_{x x}-U_{y y}\right)\right. \\
& \left.\left.-2\left(\mathbf{s} \cdot \partial_{y} \mathbf{r}\right) U_{x y}\right)\right],
\end{aligned}
$$

up to terms of quadratic order in the strains. Among functions of $\mathbf{s}$ and $\partial_{\alpha} \mathbf{r}$, other terms could be added to Eq. (19). One is an isotropic tension, $\sigma \int \mathrm{d}^{2} x U_{\alpha \alpha}$, reflecting uniform strain due to a small difference in lattice constants between monolayer and bilayer graphene. This tension can be eliminated by modifying the reference state about which strain is defined (see Refs. $[7,15,65]$ for a discussion on thermally induced uniform stretching). Such redefinition of the point of expansion implies a small shift in the elastic moduli. In addition, symmetry does not rule out a coupling of the form

$$
\frac{\lambda^{\prime}}{2}\left(U_{\alpha \alpha}\right)^{2}+\mu^{\prime} U_{\alpha \beta} U_{\alpha \beta},
$$

which contributes to the stretching elasticity of the bilayer as a whole. Due to the large difference in scale between covalent carbon-carbon interactions and interlayer van der Waals interactions, it is expected that $\lambda^{\prime}$ and $\mu^{\prime}$ are much smaller than the monolayer Lamé moduli $\lambda$ and $\mu$. Similarly, it is expected that corrections to $\lambda, \mu$, and $\kappa$ due to uniform strain are small. These effects are thus neglected in Eq. (19).

Collecting terms in Eq. (14), the model Hamiltonian for graphene bilayer thus reduces to

$$
\begin{aligned}
H= & H_{1}+H_{2}+\int \mathrm{d}^{2} x\left[\frac{g_{1}}{8 l^{4}}\left(s^{2}-l^{2}\right)^{2}\right. \\
& +\frac{g_{2}}{2 l^{2}}\left(\mathbf{s} \cdot \partial_{\alpha} \mathbf{r}\right)^{2}+\frac{g_{3}}{4 l^{2}}\left(s^{2}-l^{2}\right) U_{\alpha \alpha} \\
& \left.+\frac{g_{4}}{2 l}\left(\left(\mathbf{s} \cdot \partial_{x} \mathbf{r}\right)\left(U_{x x}-U_{y y}\right)-2\left(\mathbf{s} \cdot \partial_{y} \mathbf{r}\right) U_{x y}\right)\right] .
\end{aligned}
$$

Within the harmonic approximation, after neglection of the anisotropic term in the last line, Eq. (21) reduces to the functional derived in Ref. [39].

In analogy with the standard crystalline membrane theory, it is convenient to parametrize the coordinate vectors $\mathbf{r}(\mathbf{x})$ and $\mathbf{s}(\mathbf{x})$ by separating in-plane and out-of-plane displacement fields: $\mathbf{r}(\mathbf{x})=\left(x_{\alpha}+u_{\alpha}\right) \mathbf{v}_{\alpha}+h \mathbf{n}$ and $\mathbf{s}(\mathbf{x})=\bar{u}_{\alpha} \mathbf{v}_{\alpha}+(l+\bar{h}) \mathbf{n}$, where $\mathbf{u}, \overline{\mathbf{u}} \in \mathbb{R}^{2}, h, \bar{h} \in \mathbb{R}$. Fluctuations of relative coordinate $\bar{h}$ and the shear mode $\bar{u}_{\alpha}+l \partial_{\alpha} h$ are bounded by the couplings $g_{1}$ and $g_{2}$. For simplicity, similarly to the approach of Ref. [39], fluctuations of $\bar{h}$ and $\bar{u}_{\alpha}$ will thus be treated within a harmonic approximation. Furthermore, repeating standard approximations for single membranes $[1,4]$, we neglect the contribution $\kappa\left(\partial^{2} u_{\alpha}\right)^{2} / 2$ to the energy density and terms nonlinear in $u_{\alpha}$ in the strain tensor

$$
\begin{aligned}
U_{\alpha \beta} & =\frac{1}{2}\left(\partial_{\alpha} \mathbf{r} \cdot \partial_{\beta} \mathbf{r}-\delta_{\alpha \beta}\right) \\
& =\frac{1}{2}\left(\partial_{\alpha} u_{\beta}+\partial_{\beta} u_{\alpha}+\partial_{\alpha} h \partial_{\beta} h+\partial_{\alpha} u_{\gamma} \partial_{\beta} u_{\gamma}\right)
\end{aligned}
$$

which is thus replaced with the approximate form $u_{\alpha \beta}=$ $\frac{1}{2}\left(\partial_{\alpha} u_{\beta}+\partial_{\beta} u_{\alpha}+\partial_{\alpha} h \partial_{\beta} h\right)$.

After expansion of Eq. (21), these approximations lead to

$$
\begin{aligned}
\tilde{H}= & \int \mathrm{d}^{2} x\left[\kappa\left(\partial^{2} h\right)^{2}+\lambda\left(u_{\alpha \alpha}\right)^{2}+2 \mu u_{\alpha \beta} u_{\alpha \beta}\right. \\
& +\frac{\kappa}{4}\left(\partial^{2} \bar{h}\right)^{2}+\frac{\lambda}{4}\left(\partial_{\alpha} \bar{u}_{\alpha}\right)^{2}+\frac{\mu}{8}\left(\partial_{\beta} \bar{u}_{\alpha}+\partial_{\alpha} \bar{u}_{\beta}\right)^{2} \\
& +\frac{g_{1}}{2 l^{2}} \bar{h}^{2}+\frac{g_{2}}{2 l^{2}}\left(\bar{u}_{\alpha}+l \partial_{\alpha} h\right)^{2}+\frac{g_{3}}{2 l} \bar{h} u_{\alpha \alpha} \\
& +\frac{g_{4}}{2 l}\left(\left(\bar{u}_{x}+l \partial_{x} h\right)\left(u_{x x}-u_{y y}\right)-2\left(\bar{u}_{y}+l \partial_{y} h\right) u_{x y}\right),
\end{aligned}
$$

which will be used as a starting point in Sec. II C.

Similar to the standard theory of crystalline membranes [2,8] [see Eqs. (5)], this configuration energy possesses a continuous symmetry, which reflects the underlying invariance under rotations and translations in the embedding space: the Hamiltonian Eq. (23) is invariant under

$$
\begin{aligned}
h(\mathbf{x}) & \rightarrow h(\mathbf{x})+A_{\alpha} x_{\alpha}+B, \\
u_{\alpha}(\mathbf{x}) & \rightarrow u_{\alpha}(\mathbf{x})-A_{\alpha} h(\mathbf{x})-\frac{1}{2} A_{\alpha} A_{\beta} x_{\beta}+B_{\alpha}^{\prime}, \\
\bar{h}(\mathbf{x}) & \rightarrow \bar{h}(\mathbf{x}), \quad \bar{u}_{\alpha} \rightarrow \bar{u}_{\alpha}-l A_{\alpha},
\end{aligned}
$$

for arbitrary $A_{\alpha}, B, B_{\alpha}^{\prime}$. As in Eqs. (5), transformations with $A_{\alpha} \neq 0$ represent linearized versions of rotations in 3D space.

\section{Effective theory for flexural fluctuations}

Starting from the Gibbs probability distribution

$$
P\left[h(\mathbf{x}), u_{\alpha}(\mathbf{x}), \bar{h}(\mathbf{x}), \bar{u}_{\alpha}(\mathbf{x})\right]=\frac{1}{Z} \mathrm{e}^{-\tilde{H} / T}
$$

for fluctuations of the displacement fields $h(\mathbf{x}), u_{\alpha}(\mathbf{x}), \bar{h}(\mathbf{x})$, $\bar{u}_{\alpha}(\mathbf{x})$, we proceed to construct an effective theory describing the statistical properties of the flexural fluctuations $h(\mathbf{x})$ only, by systematically integrating out the remaining degrees of freedom. In Eq. (25), the Hamiltonian $\tilde{H}$ is assumed to be the approximate configuration energy of Eq. (23) and the normalization $Z$ is given by the partition function

$$
Z=\int\left[\mathrm{d} h \mathrm{~d} u_{\alpha} \mathrm{d} \bar{h} \mathrm{~d} \bar{u}_{\alpha}\right] \mathrm{e}^{-\tilde{H}\left[h, u_{\alpha}, \bar{h}, \bar{u}_{\alpha}\right] / T} .
$$

By explicit integration over relative fluctuations $\bar{h}$ and $\bar{u}_{\alpha}$, the effective Hamiltonian for fluctuations of $u_{\alpha}$ and $h$ fields,

$$
\tilde{H}_{\mathrm{eff}}^{\prime}\left[h(\mathbf{x}), u_{\alpha}(\mathbf{x})\right]=-T \ln \left\{\int\left[\mathrm{d} \bar{h} \mathrm{~d} \bar{u}_{\alpha}\right] \mathrm{e}^{-\tilde{H}\left[h, u_{\alpha}, \bar{h}, \bar{u}_{\alpha}\right] / T}\right\},
$$

is calculated as

$$
\begin{aligned}
\tilde{H}_{\mathrm{eff}}^{\prime}= & \int_{\mathbf{q}}\left[\frac{1}{2} \kappa_{0}(q) q^{4}|h(\mathbf{q})|^{2}+\frac{1}{2} \lambda_{0}(q)\left|u_{\alpha \alpha}(\mathbf{q})\right|^{2}\right. \\
& +\mu_{0}(q)\left|u_{\alpha \beta}(\mathbf{q})\right|^{2}+\frac{g_{4}^{2} l^{2}}{16 g_{2}^{2}}(\lambda+\mu) d_{L}(q) d_{T}(q)|A(\mathbf{q})|^{2} \\
& \left.-\frac{g_{4} l^{2}}{4 g_{2}}(\lambda+2 \mu) d_{L}(q) q^{2} h(\mathbf{q}) A^{*}(\mathbf{q})\right] .
\end{aligned}
$$

Details of the calculation are presented in the Appendix. In Eq. (28), $h(\mathbf{q})$ and $u_{\alpha \beta}(\mathbf{q})$ are Fourier components of $h(\mathbf{x})$ and 
$u_{\alpha \beta}(\mathbf{x}), A(\mathbf{q})$ is the Fourier transform of the anisotropic $D_{3 d^{-}}$ invariant field

$$
A(\mathbf{x})=\partial_{x} u_{x x}-\partial_{x} u_{y y}-2 \partial_{y} u_{x y},
$$

$\int_{\mathbf{q}}=\int \mathrm{d}^{2} q /(2 \pi)^{2}$ denotes momentum integration, and $\left|u_{\alpha \beta}(\mathbf{q})\right|^{2}=u_{\alpha \beta}(\mathbf{q}) u_{\alpha \beta}^{*}(\mathbf{q})$. Furthermore, we introduced the dimensionless functions

$$
\begin{aligned}
& d_{L}(q)=\left[1+\frac{(\lambda+2 \mu) l^{2} q^{2}}{2 g_{2}}\right]^{-1}, \\
& d_{T}(q)=\left[1+\frac{\mu l^{2} q^{2}}{2 g_{2}}\right]^{-1}, \\
& \bar{d}(q)=\left[1+\frac{\kappa l^{2} q^{4}}{2 g_{1}}\right]^{-1},
\end{aligned}
$$

and defined

$$
\begin{aligned}
& \kappa_{0}(q)=2 \kappa+\frac{(\lambda+2 \mu) l^{2}}{2} d_{L}(q), \\
& \lambda_{0}(q)=2 \lambda-\frac{g_{3}^{2}}{4 g_{1}} \bar{d}(q)+\frac{g_{4}^{2}}{4 g_{2}} d_{T}(q), \\
& \mu_{0}(q)=2 \mu-\frac{g_{4}^{2}}{4 g_{2}} d_{T}(q) .
\end{aligned}
$$

In the first three terms of Eq. (28), we recognize a Hamiltonian identical in form to the standard effective theory of crystalline membranes, Eq. (4), but with a $q$-dependent bending rigidity $\kappa_{0}(q)$ and Lamé coefficients $\lambda_{0}(q)$ and $\mu_{0}(q)$. The additional interaction involving $|A(\mathbf{q})|^{2}$ is a quadratic functional of the strain tensor and represents an anisotropic stiffness associated with gradients of the strain. Finally, the term proportional to $q^{2} h(\mathbf{q}) A^{*}(\mathbf{q})$ introduces a coupling between the component $A(\mathbf{x})$ of the gradient of strain and the local curvature $\partial^{2} h(\mathbf{x})$. In the following, these last two interactions are neglected for simplicity.

The neglection of the first of these two terms is related to the assumption that the response of the bilayer to spacedependent strain is dominated by the sum of the stiffnesses of the two isolated layers at the scales of interest. With the same assumption, we approximate

$$
\lambda_{0}(q) \simeq \lambda_{0}=2 \lambda, \quad \mu_{0}(q) \simeq \mu_{0}=2 \mu,
$$

neglecting the $q$-dependent contributions in Eq. (31). An estimate from the identification $g_{3}=2 c_{13} l$, the experimental value $c_{13}=(0 \pm 3) \mathrm{GPa}$ for graphite [66,67], and the parameters $l \simeq 3.25 \AA, g_{1} \simeq 0.8 \mathrm{eV \AA}^{-2}$ (see Sec. III) shows that the correction $-g_{3}^{2} /\left(4 g_{1}\right) \bar{d}(q)$ is much smaller than $2 \lambda$ and $2 \mu$ for any wave vector, which supports this approximation [68]. We assume that also terms $g_{4}^{2} /\left(4 g_{2}\right) d_{T}(q)$ play a minor role.

With these approximations, we are led to consider the effective Hamiltonian

$$
\tilde{H}_{\mathrm{eff}}^{\prime \prime}=\frac{1}{2} \int_{\mathbf{q}}\left[\kappa_{0}(q) q^{4}|h(\mathbf{q})|^{2}+\lambda_{0}\left|u_{\alpha \alpha}(\mathbf{q})\right|^{2}+2 \mu_{0}\left|u_{\alpha \beta}(\mathbf{q})\right|^{2}\right],
$$

which is identical in form to the standard effective theory of crystalline membranes, Eq. (4), although the bending rigidity $\kappa$ is replaced by the $q$-dependent $\kappa_{0}(q)$. The remaining integration over in-plane fields, therefore, proceeds in an usual way $[1,4,7,9,10,15]$ (see the Appendix). The resulting effective Hamiltonian for the flexural field $h(\mathbf{x})$ reads

$$
\tilde{H}_{\mathrm{eff}}=\frac{1}{2} \int_{\mathbf{q}} \kappa_{0}(q) q^{4}|h(\mathbf{q})|^{2}+\frac{1}{2} \int_{\mathbf{q}}^{\prime} Y_{0}\left|\frac{K(\mathbf{q})}{q^{2}}\right|^{2},
$$

where

$$
Y_{0}=\frac{4 \mu_{0}\left(\lambda_{0}+\mu_{0}\right)}{\lambda_{0}+2 \mu_{0}}
$$

and $K(\mathbf{q})$ is the Fourier transform of the composite field

$$
\begin{aligned}
K(\mathbf{x}) & =-\frac{1}{2}\left(\delta_{\alpha \beta} \partial^{2}-\partial_{\alpha} \partial_{\beta}\right)\left(\partial_{\alpha} h \partial_{\beta} h\right) \\
& =\frac{1}{2}\left[\left(\partial^{2} h\right)^{2}-\left(\partial_{\alpha} \partial_{\beta} h\right)\left(\partial_{\alpha} \partial_{\beta} h\right)\right],
\end{aligned}
$$

and the primed integral $\int_{\mathbf{q}}^{\prime}$ is meant to run over the nonzero wave-vector components, with the $\mathbf{q}=0$ contribution excluded $[1,7]$.

At leading order for small deformations, $K(\mathbf{x})$ coincides with the Gaussian curvature, Eqs. (13) and (34) thus expresses a long-range curvature-curvature interaction. Physically, this nonlinearity encodes a frustration of out-of-plane fluctuations due to the elastic stiffness of the layer [1,4]. Given an out-ofplane displacement field $h(\mathbf{x})$, it is not possible, in general, to choose the two displacement fields $u_{x}(\mathbf{x})$ and $u_{y}(\mathbf{x})$ in such way that the three components of the strain tensor $u_{x x}(\mathbf{x})$, $u_{y y}(\mathbf{x}), u_{x y}(\mathbf{x})$ vanish at all points. As Eq. (34) shows, regions with a finite Gaussian curvature inevitably induce a strain of order $\mathrm{O}\left(h^{2}\right)$ in the lattice, and involve an energy cost controlled by the elastic moduli $[1,4]$.

To conclude, we briefly discuss the neglected term proportional to

$$
\int_{\mathbf{q}} d_{L}(q) q^{2} h(\mathbf{q}) A^{*}(\mathbf{q})
$$

After integration over in-plane fields, this term generates an anisotropic contribution to the $q$-dependent rigidity $\kappa_{0}(q)$ of the form

$$
\delta \kappa_{0}(\mathbf{q})=-\frac{g_{4}^{2} l^{4}}{32 g_{2}^{2}} \frac{(\lambda+2 \mu)^{2}}{\mu} d_{L}^{2}(q)\left[1-\frac{\lambda+\mu}{\lambda+2 \mu} \cos ^{2}(3 \theta)\right] q^{2},
$$

where $\cos \theta=q_{x} /|\mathbf{q}|$. This contribution vanishes for $q \rightarrow 0$ and it is maximal for $q^{2} \approx 2 g_{2} /\left((\lambda+2 \mu) l^{2}\right)$, where it is of the order of $g_{4}^{2} l^{2} /\left(32 g_{2}\right)$. In addition, the term Eq. (37) generates a nonlocal interaction

$$
i \frac{(\lambda+\mu) g_{4} l^{2}}{2 g_{2}} \int_{\mathbf{q}}^{\prime} d_{L}(q) q_{x}\left(q_{x}^{2}-3 q_{y}^{2}\right) h(\mathbf{q}) \frac{K^{*}(\mathbf{q})}{q^{2}},
$$

which couples the curvature tensor $\partial_{\alpha} \partial_{\beta} h$ to the approximate Gaussian curvature $K(\mathbf{x})$. Consideration of these effects is beyond the scope of this paper. It is expected that the term Eq. (37) does not modify the exponent of the scaling behavior.

\section{MODEL PARAMETERS FOR AB-STACKED BILAYER GRAPHENE}

As discussed above, the bending rigidity $\kappa$ and the Lamé coefficients $\lambda$ and $\mu$ are approximated by their values for monolayer graphene, which is justified by the weakness of van der Waals interactions in comparison with in-plane bonding. 
TABLE I. Parameters for bilayer graphene obtained from first-principle calculations, compared with the elastic constants of AB-stacking graphite reported in Ref. [66]. In the elastic moduli of graphite, results in brackets were calculated considering van der Waals corrections [66]. The lattice constant $a$ and interlayer distance $l$ are expressed in $\AA$, the couplings $g_{1}$ and $g_{2}$ in eV $\AA^{-2}$, and the elastic moduli $c_{33}, c_{44}$ in GPa. In the case of graphite, the values of $g_{1}$ and $g_{2}$ in the table are defined by the identifications $g_{1} \equiv c_{33} l, g_{2} \equiv c_{44} l$, where $l$ is the graphite interlayer distance.

\begin{tabular}{lcccccc}
\hline \hline & $a$ & $l$ & $g_{1}$ & $g_{2}$ & $c_{33}$ & $c_{44}$ \\
\hline Bilayer & 2.46 & 3.2515 & 0.80 & 0.11 & - \\
Graphite & 2.45 & $3.42 \pm 0.01$ & $0.62(0.90)$ & $0.096(0.10)$ & $29(42)$ & $4.5(4.8)$ \\
\hline \hline
\end{tabular}

In the case of the in-plane Young modulus $Y$, this approximation is consistent with experimental values illustrated in Ref. [37], which indicate for bilayer graphene a value of $Y$ approximately equal to twice the corresponding monolayer modulus.

The elastic moduli and the bending stiffness of a monolayer graphene have been investigated extensively (see, e.g., Refs. [37,43,69,70]). Theoretical predictions and estimates of $\kappa$ lead to values between $0.69 \mathrm{eV}$ and approximately $2.4 \mathrm{eV}$ [69-71].

By comparing results of atomistic Monte Carlo simulations and continuum membrane theory, the bare bending rigidity $\kappa$ was predicted to present a significant temperature dependence [19]. This was attributed to anharmonic interactions between acoustic modes and other phonon branches or, more generally, with degrees of freedom not captured by the membrane model. In Ref. [34], a similar result was obtained for bilayer graphene. In addition, by a similar fitting, method Ref. [34] determined temperature dependencies of the interlayer compression modulus, analog to $g_{1}$ in Eq. (23).

In the following, we neglect these temperature dependencies and, similarly, effects of thermal expansion on the lattice constant $a$ and the interlayer distance $l$. In further calculations, we adopt the values $\lambda \simeq 3.8 \mathrm{eV} \AA^{-2}$ and $\mu \simeq 9.3 \mathrm{eV}$ $\AA^{-2}$, which we deduced from the first-principles results of Ref. [72], and assume $\kappa=1 \mathrm{eV}[21,22]$.

To determine interlayer coupling parameters $g_{1}$ and $g_{2}$, we have performed density-functional theory calculations on AB-stacked bilayer graphene [see Figs. 1(a) and 1(b)]. We use the plane-wave-based code PWSCF as implemented in the QUANTUM ESPRESSO ab initio package [73]. A vacuum layer of more than $15 \AA$ has been added to avoid perpendicular interaction between neighboring cells. The quasi-Newton algorithm for ion relaxation is applied until the components of all forces are smaller than $10^{-5} \mathrm{Ry} /$ bohr. The interlayer distance $l$ and the lattice parameter $a$ obtained after relaxation are shown in Table I. For the self-consistent calculations, we use a $36 \times 36 \times 1$ grid. The kinetic energy cutoff is set to 100 Ry. Projector augmented wave pseudopotentials within the Perdew-Burke-Ernzerhoff approximation [74] for the exchange-correlation functional are used for the $\mathrm{C}$ atoms. Van der Waals dipolar corrections are introduced during relaxation through the Grimme-D2 model [75].

To calculate the interlayer shear modulus $g_{2}$ and the outof-plane compression modulus $g_{1}$, we apply deformations as shown in Figs. 2(a) and 2(b), respectively, to the bilayer graphene unit cell. For simplicity, a frozen-ion approximation is assumed: During deformation, all atoms are displaced rigidly without allowing for a relaxation of the internal struc- ture of the unit cell. After application of a sequence of relative shifts $\delta x$ between carbon layers and variations $\delta l$ of the layer-to-layer distance, the total energy per unit area $E / A$ is fitted as

$$
\begin{aligned}
& \frac{E}{A}=\frac{E_{0}}{A}+\frac{g_{1}}{2} \frac{\delta l^{2}}{l^{2}}, \\
& \frac{E}{A}=\frac{E_{0}}{A}+\frac{g_{2}}{2} \frac{\delta x^{2}}{l^{2}} .
\end{aligned}
$$

The resulting values for $g_{1}$ and $g_{2}$ are illustrated in Table I.

It is natural to compare the values of $g_{1}$ and $g_{2}$ with corresponding 3D elastic moduli in graphite. A stack of membranes with interactions of the form Eq. (23) between nearest-neighboring layers and vanishing interactions between non-neighboring layers exhibits 3D elastic moduli $c_{33}=g_{1} / l$ and $c_{44}=g_{2} / l$, where $c_{33}$ and $c_{44}$ are defined according to the Voigt notation:

$$
\begin{aligned}
\frac{E}{V} & =\frac{1}{2} \sum_{i, j} c_{i j} \epsilon_{i} \epsilon_{j}, \quad u_{\alpha \beta}=\left[\begin{array}{lll}
u_{x x} & u_{x y} & u_{x z} \\
u_{y x} & u_{y y} & u_{y z} \\
u_{z x} & u_{z y} & u_{z z}
\end{array}\right] \\
& =\left[\begin{array}{ccc}
\epsilon_{1} & \frac{\epsilon_{6}}{2} & \frac{\epsilon_{5}}{2} \\
\frac{\epsilon_{6}}{2} & \epsilon_{2} & \frac{\epsilon_{4}}{2} \\
\frac{\epsilon_{5}}{2} & \frac{\epsilon_{4}}{2} & \epsilon_{3}
\end{array}\right]
\end{aligned}
$$

where $E / V$ is the energy density of the 3D solid under uniform strain. In Table I, our results for bilayer graphene are compared with $a b$ initio calculations for ideal AB-stacking graphite reported in Ref. [66]. The comparison indicates that values of $g_{1}$ and $g_{2}$ calculated in this work are of the same order of the corresponding graphite stiffnesses. We note, however, that the exact value of the shear modulus in multilayer graphene is still far from being understood. Reported values for the interlayer shear modulus exhibit a large dispersion (see, e.g., Refs. [37,46]). Raman measurements give values of the order of 4-5 GPa, while direct measurements using (a) Shear strain

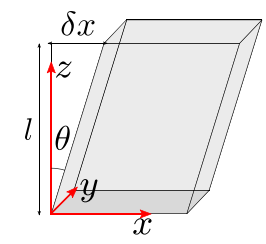

(b) Compression/stretch

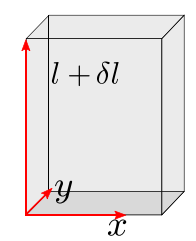

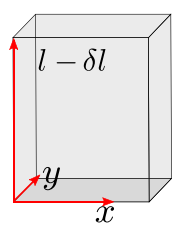

FIG. 2. Scheme of the shear and out-of-plane strains. 


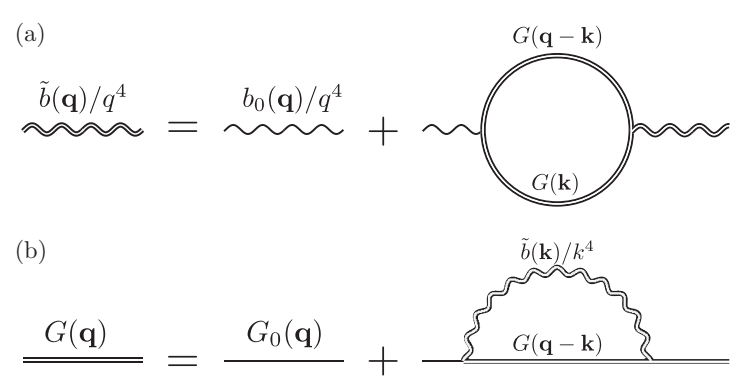

FIG. 3. Graphical representation of the self-consistent screening approximation.

mechanical approaches give values of $0.36-0.49 \mathrm{GPa}$, increasing with the number of layers. This big discrepancy calls for a better understanding of interlayer dipolar or van der Waals interactions in layered materials, which is beyond the scope of this work. Experimental values of the interlayer shear modulus in graphite also exhibit a large scatter [37].

\section{SELF-CONSISTENT SCREENING APPROXIMATION}

Equilibrium correlation functions of the flexural field $h(\mathbf{x})$ at a temperature $T$ can be calculated by functional integration from the effective Hamiltonian $\tilde{H}_{\text {eff }}$, Eq. (34). In this work, the two-point correlation function $G(\mathbf{q})=\left\langle|h(\mathbf{q})|^{2}\right\rangle$ is calculated within the SCSA $[9,10,51]$.

In the considered model for bilayer graphene, the problem differs from conventional membrane theory only by the $q$-dependence of $\kappa_{0}(q)$. Therefore, SCSA equations can be written in a standard way [10], by adapting the conventional equations with the replacements $\kappa \rightarrow \kappa_{0}(q), Y \rightarrow Y_{0}$.

The SCSA is defined diagrammatically in Fig. 3: By neglecting vertex corrections, Dyson equations are truncated to a closed set of integral equations for $G(\mathbf{q})$ and a screenedinteraction propagator $D(\mathbf{q})$. For physical 2D membranes in 3D space, SCSA equations read [10]

$$
\begin{gathered}
G^{-1}(\mathbf{q})=G_{0}^{-1}(\mathbf{q})+\Sigma(\mathbf{q}), \\
\tilde{b}^{-1}(\mathbf{q})=b_{0}^{-1}(\mathbf{q})+3 I(\mathbf{q}),
\end{gathered}
$$

where the self-energy $\Sigma(\mathbf{q})$ and the polarization bubble $I(\mathbf{q})$ are, respectively,

$$
\Sigma(\mathbf{q})=2 \int_{\mathbf{k}}\left[q^{2} k^{2}-(\mathbf{q} \cdot \mathbf{k})^{2}\right]^{2} \frac{\tilde{b}(\mathbf{k})}{k^{4}} G(\mathbf{q}-\mathbf{k})
$$

and

$$
I(\mathbf{q})=\frac{1}{3 q^{4}} \int_{\mathbf{k}}\left[q^{2} k^{2}-(\mathbf{q} \cdot \mathbf{k})^{2}\right]^{2} G(\mathbf{q}-\mathbf{k}) G(\mathbf{k}) .
$$

For membranes described by Eq. (4), the zero-order propagators are

$$
G_{0}^{-1}(\mathbf{q})=\frac{\kappa q^{4}}{T}, \quad b_{0}(\mathbf{q})=\frac{Y}{2 T} .
$$

For bilayer graphene, after the approximations $\lambda_{0}(q) \simeq 2 \lambda$ and $\mu_{0}(q) \simeq 2 \mu$ (see Sec. II C), the zero-order flexural-field and interaction propagators for bilayer graphene read

$$
G_{0}^{-1}(\mathbf{q})=\frac{\kappa_{0}(q) q^{4}}{T}, \quad b_{0}(\mathbf{q})=\frac{Y_{0}}{2 T},
$$

where, as in Eq. (31),

$$
\kappa_{0}(q)=2 \kappa+\frac{(\lambda+2 \mu) l^{2}}{2}\left[1+\frac{(\lambda+2 \mu) l^{2} q^{2}}{2 g_{2}}\right]^{-1} .
$$

In the long-wavelength limit, identification of power-law solutions of SCSA equations within the strong-coupling assumption $\Sigma(\mathbf{q}) \gg G_{0}^{-1}(\mathbf{q}), I(\mathbf{q}) \gg b_{0}^{-1}(\mathbf{q})$ yields analytical equations for the universal exponent $\eta$. After generalization to a theory of $D$-dimensional membranes embedded in a $\left(D+d_{c}\right)$-dimensional ambient space, the SCSA exponent $\eta\left(D, d_{c}\right)$ is exact to first order in $\varepsilon=4-D$, to leading order in a $1 / d_{c}$-expansion and for $d_{c}=0[9,10]$. For the physical case $D=2, d_{c}=1$, the SCSA exponent $\eta=4 /(1+$ $\sqrt{15}) \simeq 0.821$, shows a good agreement with complementary approaches such as numerical simulations and the nonperturbative renormalization group [11]. As compared with the SCSA, a second-order generalization which includes dressed diagrams with the topology of $\mathrm{O}\left(1 / d_{c}^{2}\right)$ graphs in a large$d_{c}$ expansion, leads to quantitatively small corrections to universal quantities for $D=2, d_{c}=1$ [51], which supports the accuracy of the method. Recently, SCSA predictions have been compared with exact analytical calculations of $\eta$ in second-order large- $d_{c}[76]$ and $\varepsilon$-expansions $[17,18]$. In Ref. [17], it was shown that the SCSA equations are exact at $\mathrm{O}\left(\varepsilon^{2}\right)$ within a nonstandard dimensional continuation of the theory to arbitrary $D$. A more general two-loop theory was developed in Ref. [18], where a larger space of theories was considered. For models equivalent to the conventional dimensionally continued membrane theory, the $\mathrm{O}\left(\varepsilon^{2}\right)$ was shown to deviate from the SCSA prediction.

To determine correlation functions at an arbitrary wave vector $q$, we solve SCSA equations numerically by an iterative algorithm. Starting from noninteracting propagators $G(\mathbf{q})=G_{0}(\mathbf{q}), \tilde{b}(\mathbf{q})=b_{0}(\mathbf{q})$, Eqs. (45) and (46) are used to determine the zero-order polarization bubble $I(\mathbf{q})$ and the first approximation to the screened interaction $\tilde{b}_{1}(\mathbf{q})$. The self-energy diagram in Fig. 3(b) is then calculated as a loop integral of $\tilde{b}_{1}(\mathbf{q})$ and $G_{0}(\mathbf{q})$, leading to a dressed Green's function $G_{1}(\mathbf{q})$. Iteration of the process generates a sequence of screened functions and dressed propagators,

$$
\begin{aligned}
G_{n+1}^{-1}(\mathbf{q}) & =G_{0}^{-1}(\mathbf{q})+\Sigma_{n}(\mathbf{q}), \\
\tilde{b}_{n+1}^{-1}(\mathbf{q}) & =b_{0}^{-1}(\mathbf{q})+3 I_{n}(\mathbf{q}), \\
\Sigma_{n}(\mathbf{q}) & =2 \int_{\mathbf{k}}\left[q^{2} k^{2}-(\mathbf{q} \cdot \mathbf{k})^{2}\right]^{2} \frac{\tilde{b}_{n+1}(\mathbf{k})}{k^{4}} G_{n}(\mathbf{q}-\mathbf{k}), \\
I_{n}(\mathbf{q}) & =\frac{1}{3 q^{4}} \int_{\mathbf{k}}\left[q^{2} k^{2}-(\mathbf{q} \cdot \mathbf{k})^{2}\right]^{2} G_{n}(\mathbf{q}-\mathbf{k}) G_{n}(\mathbf{k}),
\end{aligned}
$$

which, after convergence, approach solutions to the SCSA equations. At each step in the iteration process, correlation functions are calculated on a grid of 50 wave-vector points, evenly spaced in logarithmic scale and ranging between $10^{-7} \AA^{-1}$ and $110 \AA^{-1}$. Calculations with grids of 26 and 29 points are also performed to estimate the numerical accuracy [77].

Twenty-five steps of the iteration algorithm are illustrated in Fig. 4. To calculate loop integrals, at each iteration $G(\mathbf{q})$ and $\tilde{b}(\mathbf{q})$ are interpolated by cubic splines [78] in logarithmic scale: $G(\mathbf{q})$ and $\tilde{b}(\mathbf{q})$ are interpolated as $G_{n}(\mathbf{q})=$ 


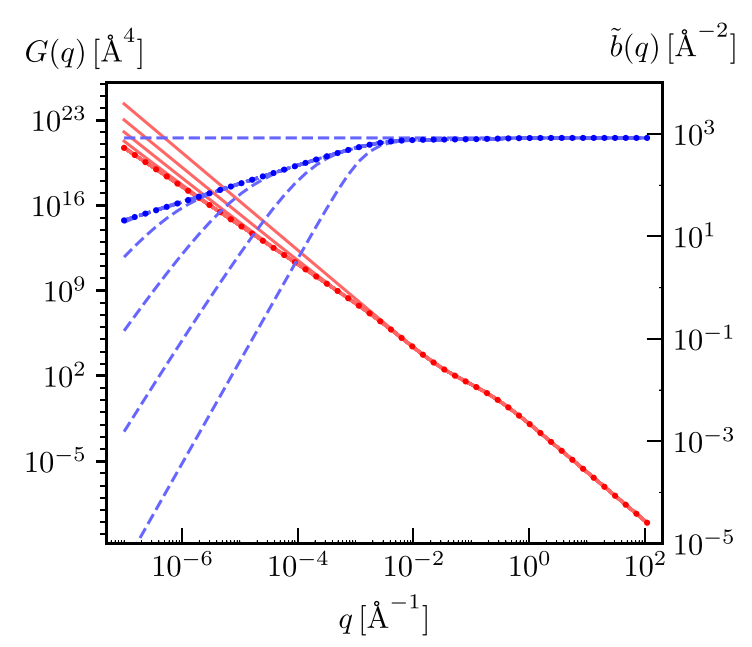

FIG. 4. Sequence of correlation functions (red solid lines) and screened interactions (blue dashed lines) obtained by 25 iterations of the convergence algorithm. Data in the graph refer to a bilayer membrane with the parameters $\lambda=3.8 \mathrm{eV} \AA^{-2}, \mu=9.3 \mathrm{eV}^{-2}$, $\kappa=1 \mathrm{eV}, l=3.2515 \AA, g_{2}=0.11 \mathrm{eV} \AA^{-2}$, and $T=300 \mathrm{~K}$. Correlation functions evaluated at the last iteration on the 50-point grid are shown by dots.

$A_{1} \exp \left[f_{1}(\ln (q / B))\right], \tilde{b}_{n}(\mathbf{q})=A_{2} \exp \left[f_{2}(\ln (q / B))\right]$, where $f_{1}$ and $f_{2}$ are cubic splines and $A_{1}, A_{2}, B$ are constants. In the region $q<10^{-7} \AA^{-1}$, which is not covered by the wavevector grid, functions are extrapolated as pure power laws, $G_{n} \propto q^{-\eta(n)}$ and $\tilde{b}_{n} \propto q^{\eta_{u}(n)}$ with exponents and amplitudes matching the first two points in the grid.

In the calculation of integrals, we split $2 \mathrm{D}$ wave-vector integration into a sequence of 1D integrals over $k_{y}$ and $k_{x}$, the components of $\mathbf{k}$, respectively, transverse and longitudinal to the external wave vector q. In the computation, we use an adaptive algorithm for single-variable integration [78], and include $k_{y}$ integration in the function called by the outer $k_{x}$ integral. Inner and outer integrals are evaluated within a relative accuracy $1.49 \times 10^{-8}$ and $10^{-7}$, respectively.

Although the self-energy and polarization bubble are convergent, a hard ultraviolet cutoff $\Lambda=100 \AA^{-1}$ is imposed in explicit calculations. To estimate the numerical error due to the finite UV cutoff, we compared data sets calculated with $\Lambda=100 \AA^{-1}$ and $\Lambda=1000 \AA^{-1}$, which were obtained by calculating numerical solutions on wave-vector grids consisting of 26 and 29 points respectively. Upon this change in UV cutoff, data sets for $G(q)$ and $\tilde{b}(q)$ deviate by less than $10^{-5}$ [77].

In the numerical calculations, difficulties stem from the rapid variation of functions in regions of much smaller size than the integration domain and from the slow decay of integration tails at large $k$. To address these problems, integrals are performed piecewise. Specifically, the $k_{y}$ integration domain is split into contiguous intervals with extrema $\left\{0,10^{-1} q_{1}, q_{1}, 10 q_{1}, q_{2}, 10 q_{2}, 10^{2} q_{2}, 10^{3} q_{2}\right\}$, where $q_{1}=\sqrt{q|\mathbf{q}-\mathbf{k}|}$ and $q_{2}=\max [q,|\mathbf{q}-\mathbf{k}|]$. For any $q$ and $k_{x}$ and at any steps in the iteration process, characteristic scales $q_{1}$ and $q_{2}$ define roughly the width in $k_{y}$ integration which contributes mostly to the integral value. The piecewise calculation defined above is then able to capture a small-scale peak in the integrand function and a long tail for $k_{y} \gg q_{2}$. In the subsequent $k_{x}$ integrations, similarly, subintervals are chosen as $\left\{. .,-10 q,-q, 0, q, 10 q, 10^{2} q ..\right\}$.

After 25 iterations of the algorithm, the values of $G_{n}(\mathbf{q})$ and $\tilde{b}_{n}(\mathbf{q})$ at the grid of sampled wave-vector points converge within a relative deviation smaller than $10^{-10}$. The final results (see Sec. V) reproduce the analytically known SCSA exponent and amplitude ratio [9,10,51] closely: An estimate of the exponents $\eta, \eta_{u}$ and the amplitudes $z_{1}, z_{2}$ of the scaling behavior

$$
G^{-1}(\mathbf{q})=z_{1} q^{4-\eta}, \quad \tilde{b}(\mathbf{q})=z_{2} q^{\eta_{u}},
$$

from the first two points of the wave-vector grid gives values in the range $\eta=0.8208515 \div 0.8208524$, $\eta_{u}=0.35829478 \div 0.35829524$, and $z_{1}^{2} / z_{2}=0.1781321 \div$ 0.1781381 for considered data sets for monolayer graphene at $T=300 \mathrm{~K}$ and bilayer graphene at different temperatures between 10 and $1500 \mathrm{~K}$. These results are in close agreement with the analytical predictions $\eta=4 /(1+\sqrt{15}) \simeq$ $0.82085238, \eta_{u}=2-2 \eta \simeq 0.35829523$, and [10,51]

$$
\frac{z_{1}^{2}}{z_{2}}=\frac{3}{16 \pi} \frac{\Gamma^{2}(1+\eta / 2) \Gamma(1-\eta)}{\Gamma^{2}(2-\eta / 2) \Gamma(2+\eta)} \simeq 0.17813212 \ldots
$$

The individual amplitudes $z_{1}$ and $z_{2}$ and the crossover behaviors at finite $q$ are more sensitive to numerical error. A limitation to numerical accuracy derives from the need to interpolate $G(q)$ and $\tilde{b}(q)$ from a discrete set of data points. To estimate the order of the corresponding error, the numerical solution of SCSA equations was repeated after reduction to a broader grid, consisting of 26 wave-vector points. Compared to data evaluated with the $50 q$-point grid, interpolating functions exhibit a maximum relative deviation of the order of $2 \%$ in all considered sets of data (see Ref. [77] for a more detailed analysis). The amplitudes $z_{1}$ and $z_{2}$ of the long-wavelength scaling regime exhibit a smaller discrepancy, of the order of $10^{-3}$, upon change from the finer to the broader wave-vector grid.

Numerical results indicate that the numerical values of the exponent and the amplitude ratio $z_{1}^{2} / z_{2}$ are much more accurate than the numerical precision in calculations of nonuniversal properties such as the amplitude and finitewavelength dependencies of $G(q)$ and $\tilde{b}(q)$. Qualitatively, universal properties are only sensitive to the region of small momenta, where $G(q)$ and $\tilde{b}(q)$ approach pure powers and the precision of numerical interpolation improves significantly.

\section{RESULTS}

The numerical algorithm described in Sec. IV was used to determine solutions to the SCSA equations for graphene monolayer and bilayers at temperatures $T=10,300$, and 1500 K. Results are illustrated in Figs. 5-8, while numerical data are reported in Ref. [77].

All reported results are derived within the framework of continuum models discussed in Sec. II, which do not capture the effects of discreteness of the lattice. Figures 5-8 illustrate correlation functions in the full wave-vector range employed for the numerical calculation of the continuum-limit solution, $10^{-7} \AA^{-1}<q<10^{2} \AA^{-1}$, although, on the lattice, only degrees of freedom with $q \lesssim 1 \AA^{-1}$ are physical. 


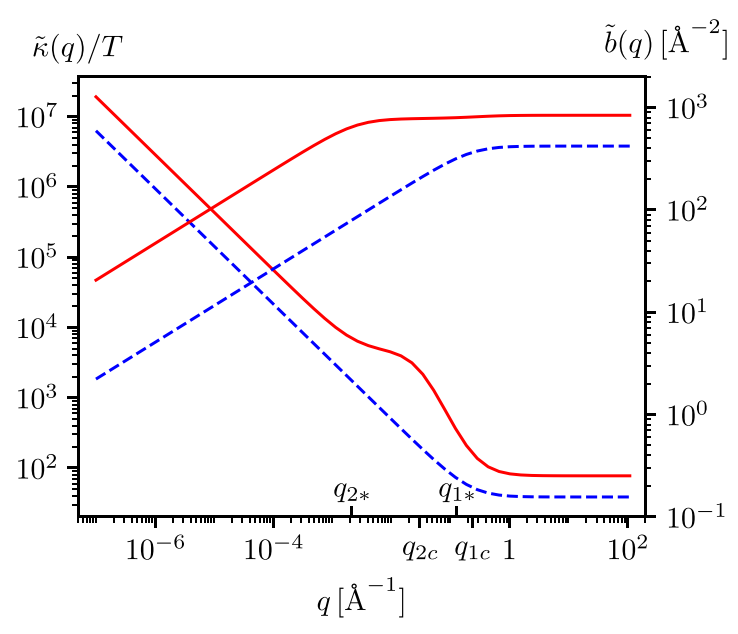

FIG. 5. Renormalized bending rigidity $\tilde{\kappa}(q)=T G^{-1}(q) / q^{4}$ and renormalized elastic coefficient $\tilde{b}(q)$ for continuum models of monolayer (blue dashed lines) and bilayer graphene (red solid lines) at $T=300 \mathrm{~K}$. For $q \rightarrow 0, \tilde{\kappa}(q)$ diverges for both curves as $q^{-\eta}$ and $\tilde{b}(q)$ approaches 0 as $q^{2-2 \eta}$.

The renormalized bending rigidity $\tilde{\kappa}(q) \equiv T G^{-1}(q) / q^{4}$, and the renormalized elastic modulus $\tilde{b}(q)[9,10]$ for singlelayer graphene at room temperature are illustrated by blue dashed lines in Fig. 5. As it is completely general within the framework of the elasticity model, Eq. (4), interaction effects are weak for $q \gtrsim q_{*}$, where $q_{*}=\sqrt{3 T Y /\left(16 \pi \kappa^{2}\right)}[10,19]$. In the limit $q \gg q_{*}, \tilde{b}(q)$ and $\tilde{\kappa}(q)$ approach their bare values

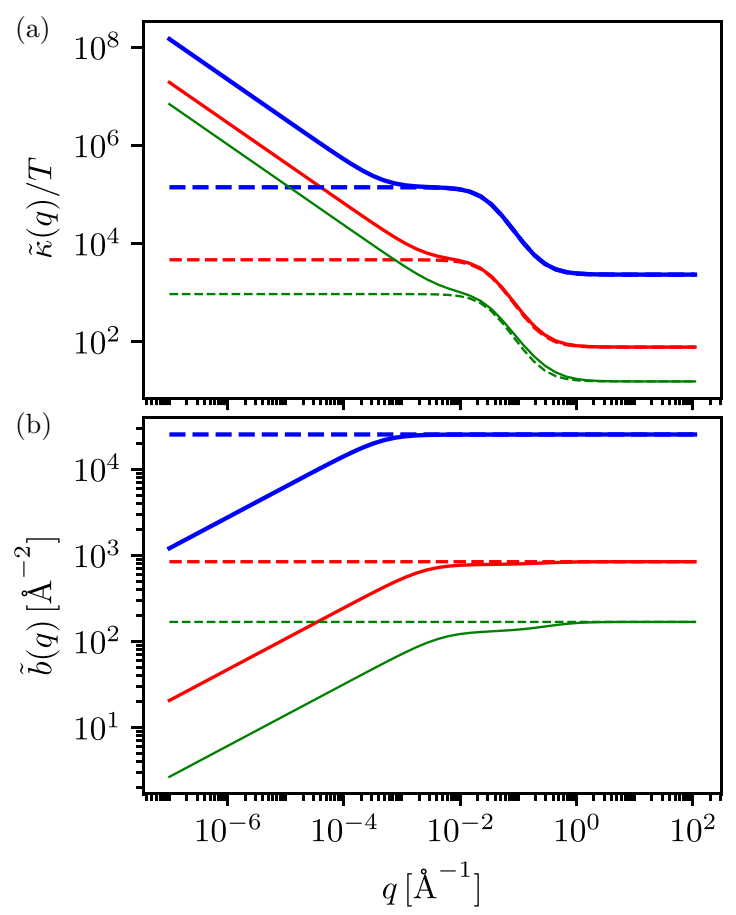

FIG. 6. (a) Renormalized bending rigidity and (b) renormalized elastic modulus for bilayer graphene at $T=10 \mathrm{~K}$ (thick blue lines), $300 \mathrm{~K}$ (intermediate red lines), and $1500 \mathrm{~K}$ (thin green lines). Dashed lines illustrate the corresponding functions in the harmonic approximation.

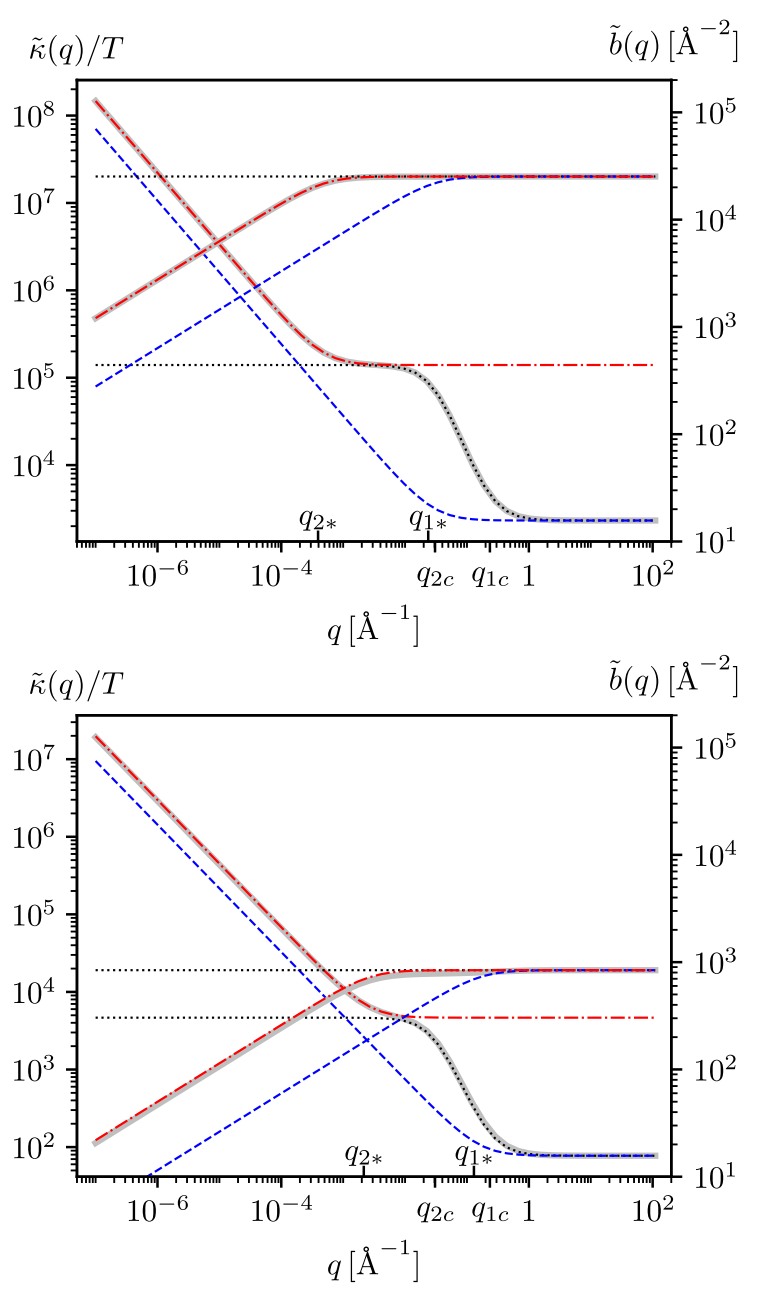

FIG. 7. Renormalized bending rigidity and renormalized elastic modulus for bilayer graphene at $T=10 \mathrm{~K}$ (top panel) and $T=$ $300 \mathrm{~K}$ (bottom panel). Thick solid grey lines represent $\tilde{\kappa}(q) / T$ and $\tilde{b}(q)$ obtained by numerical solution of SCSA equations for bilayer graphene. The corresponding functions in the harmonic approximation $\kappa_{0}(q)$ and $b_{0}(q)=b_{0}=Y_{0} /(2 T)$ are illustrated as black dotted lines. The blue dashed curves show the SCSA correlation functions for a single membrane with Young modulus $2 Y$ and bending rigidity $2 \kappa$, i.e., twice as large than in monolayer graphene. The correlation functions of a single membrane with Young modulus $2 Y$ and the much larger bending rigidity $2 \kappa+(\lambda+2 \mu) l^{2} / 2$ is illustrated by red dash-dotted lines.

$Y /(2 T)$ and $\kappa$, with negligible renormalizations. In contrast, for $q \lesssim q_{*}$ a strong coupling regime sets in. For $q \ll q_{*}$ the self-energy $\Sigma(q)$ and the polarization function $I(q)$ are much larger than the harmonic propagators $G_{0}^{-1}(q)$ and $b_{0}^{-1}(q)$; correlation functions scale as power laws $[9,10,51]$ :

$$
G^{-1}(\mathbf{q})=z_{1} q^{4-\eta}, \quad \tilde{b}(\mathbf{q})=z_{2} q^{\eta_{u}} .
$$

As mentioned above, numerical results are in close agreement with the scaling relation $\eta_{u}=2-2 \eta$, and the predictions, exact within SCSA, $\eta=4 /(1+\sqrt{15})$ and $z_{1}^{2} / z_{2} \simeq$ $0.17813212[9,10,51]$.

By a simple rescaling, the numerical solution obtained for monolayer graphene can be adapted to any membrane described by the elasticity model, Eq. (4). For any such mem- 

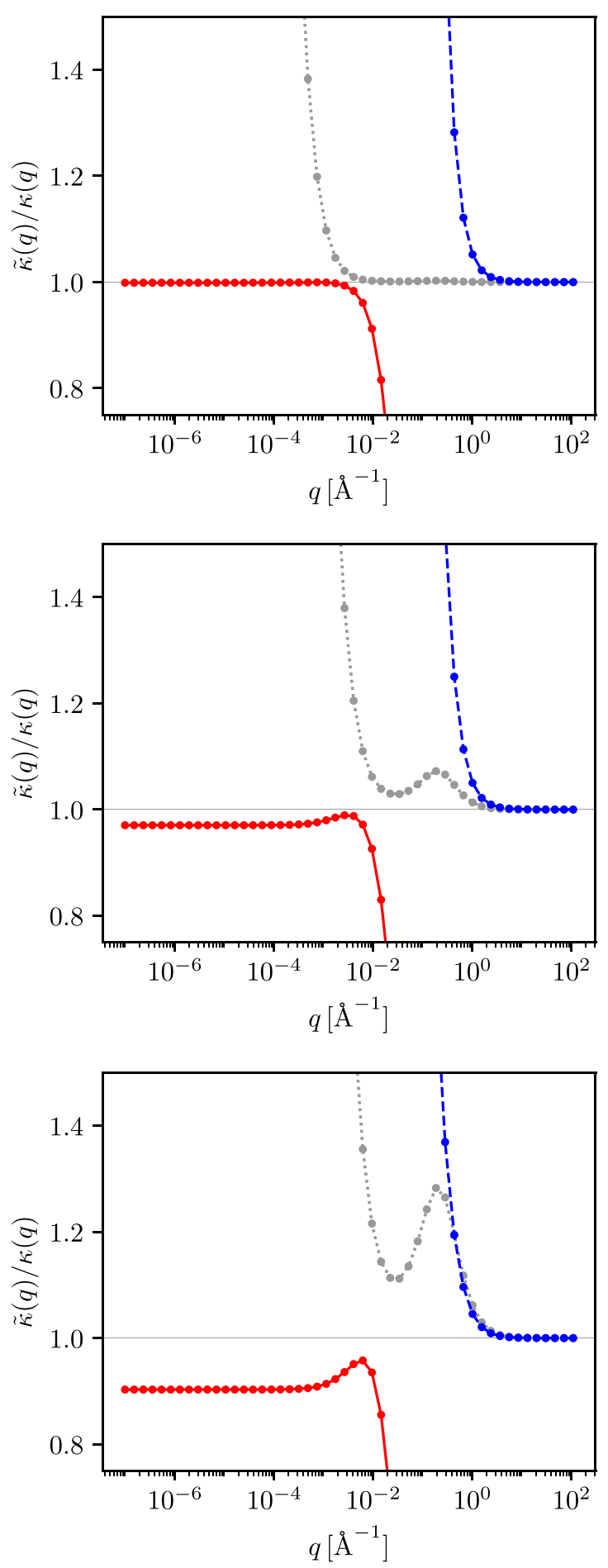

FIG. 8. Ratio between the renormalized bending rigidity $\tilde{\kappa}(q)$ and the bare effective rigidity $\kappa_{0}(q)$ (grey dotted lines), the renormalized rigidity $\tilde{\kappa}_{1}(q)$ of a single-layer membrane with parameters $2 Y$ and $2 \kappa$ (blue dashed line), and the analog function $\tilde{\kappa}_{2}(q)$ for parameters $2 Y$ and $\bar{\kappa}_{0}$ (red solid line). Panels (a)-(c) refer to data at $T=10,300$, and $1500 \mathrm{~K}$, respectively. A horizontal line at 1 is drawn as guide to the eye.

brane, the statistics of out-of-plane fluctuations is governed by a Hamiltonian of the form (34) with a wave-vectorindependent rigidity $\kappa_{0}(q)=\kappa$ and Young modulus $Y_{0}(q)=$
$Y$. A scaling analysis then shows that

$$
G(\mathbf{q})=\frac{T}{\kappa q^{4}} g\left(\frac{q}{q_{*}}\right)
$$

and

$$
\tilde{b}(\mathbf{q})=b_{0} f\left(\frac{q}{q_{*}}\right)=\frac{Y}{2 T} f\left(\frac{q}{q_{*}}\right),
$$

where $g(x)$ and $f(x)$ are independent of temperature and elastic parameters. In particular, the coefficient $z_{1}$ governing the amplitude of the scaling behavior has the form [79]

$$
z_{1}=\bar{z}_{1} \frac{\kappa q_{*}^{\eta}}{T},
$$

where $\bar{z}_{1}$ is independent of $T, \kappa$, and $Y$. An estimate from the amplitude of $G$ in monolayer graphene gives $\bar{z}_{1} \simeq 1.177$ within SCSA. In the following, the scaling-analysis relations Eqs. (53) and (54) are used to convert numerical data collected for monolayer graphene at $T=300 \mathrm{~K}$ to single membranes with arbitrary elastic parameters and temperature.

As Figs. 5-7 show, correlation functions in bilayer graphene exhibit a more intricate crossover behavior which extends from microscopic to mesoscopic scales. In contrast with the monolayer elasticity theory, the behavior of a bilayer is controlled by several length scales. The effective bare bending rigidity $\kappa_{0}(q)$, Eq. (48), approaches limiting values $2 \kappa$ and $\bar{\kappa}_{0}=2 \kappa+(\lambda+2 \mu) l^{2} / 2$ for $q \gtrsim q_{1 c}$ and for $q \lesssim q_{2 c}$ respectively, where

$$
q_{1 c}=\sqrt{\frac{g_{2}}{2 \kappa}} \simeq 0.2 \AA^{-1}
$$

and

$$
q_{2 c}=\frac{1}{l} \sqrt{\frac{2 g_{2}}{\lambda+2 \mu}} \simeq 3 \times 10^{-2} \AA^{-1} .
$$

A crossover in the mechanical behavior [39] takes place between these two scales: $q_{2 c}<q<q_{1 c}$. The strong $q$ dependence of $\kappa_{0}(q)$ has a crucial impact on the harmonic correlation functions. The effective rigidity $\tilde{\kappa}(q)=T G^{-1}(q) / q^{4}$ and elastic coefficient $\tilde{b}(q)=b_{0}$ in the harmonic approximation, which coincide with their bare value $\kappa_{0}(q)$ and $b_{0}(q)=$ $b_{0}$, are illustrated by dashed lines in Fig. 6 and by grey dotted lines in Fig. 7.

At finite temperatures, for a single membrane, crossover from weak to strong coupling is marked by the Ginzburg scale $q_{*}=\sqrt{3 T Y /\left(16 \pi \kappa^{2}\right)}$. In the case of bilayer graphene, two scales analog to $q_{*}$ can be anticipated:

$$
q_{1 *}=\sqrt{\frac{3 T}{16 \pi} \frac{(2 Y)}{(2 \kappa)^{2}}}=\frac{q_{*}}{\sqrt{2}}
$$

and

$$
q_{2 *}=\sqrt{\frac{3 T}{16 \pi} \frac{(2 Y)}{\bar{\kappa}_{0}^{2}}} .
$$

While $q_{1 *}$ is close to the Ginzburg scale for a monolayer graphene, $q_{2 *}$ is smaller by two orders of magnitude due to the strong enhancement of $\bar{\kappa}_{0} \gg 2 \kappa$. 
The inverse lattice spacing $1 / a \simeq 1 \AA^{-1}$ defines a further scale for fluctuations of the atomic crystal, which marks a limit of validity for the continuum model employed here.

To study the interplay and overlap between these crossover effects, we analyzed fluctuations in bilayer graphene at temperatures $T=10,300$, and $1500 \mathrm{~K}$. For small temperatures, the mechanical and the weak-strong coupling crossovers are disentangled. At $T=10 \mathrm{~K}$ both $q_{2 *} \simeq 4 \times 10^{-4} \AA^{-1}$ and $q_{1 *} \simeq 2 \times 10^{-2} \AA^{-1}$ are smaller than $q_{1 c}$, and furthermore $q_{2 *} \ll q_{2 c}$. As confirmed by the numerical results, throughout the region $q_{2 c}<q<q_{1 c}$ thermal effects are negligible. Strong coupling behavior sets in only at $q<q_{2 *}<q_{2 c}$, a region where $\kappa_{0}(q)$ has already converged to its limiting value $\bar{\kappa}_{0}$. A more detailed analysis of the collected numerical data shows that for $q>4 \times 10^{-3} \AA^{-1}, \tilde{\kappa}(q)=T G^{-1}(q) / q^{4}$ and $\tilde{b}(q)$ differ from their harmonic approximations $\kappa_{0}(q)$ and $b_{0}(q)$ by less than $3 \%$. For $q<4 \times 10^{-3} \AA^{-1}$, instead, numerical data agree within $3 \%$ with correlation functions of a single membrane with Young modulus $2 Y$ and rigidity $\bar{\kappa}_{0}$, which was obtained by rescaling monolayer graphene results via Eqs. (53) and (54). In particular, in the scaling region $q \ll q_{2 *}$, the amplitude $z_{1}^{\prime}$ of the power-law behavior $G^{-1}(\mathbf{q})=z_{1}^{\prime} q^{4-\eta}$ differs from the corresponding single-membrane value

$$
z_{1}=\bar{z}_{1} \frac{\bar{\kappa}_{0} q_{2 *}^{\eta}}{T} \simeq 1.177 \frac{\bar{\kappa}_{0} q_{2 *}^{\eta}}{T}
$$

only by a deviation of the order of $10^{-3}$.

Figure 7 illustrates an explicit comparison between full correlation functions for bilayer graphene at $T=10 \mathrm{~K}$, their harmonic approximation, and the corresponding functions for single membranes having Young modulus $2 Y$ and bending rigidity $2 \kappa$ and $\bar{\kappa}_{0}$. Ratios between corresponding functions are presented in Fig. 8.

At room temperature, the mechanical and the weak-strong coupling crossovers have a more sizable overlap: The characteristic scale $q_{1 *} \simeq 0.13 \AA^{-1}$ is of the same order of $q_{1 c}$. As can be seen in Fig. 8(b), the renormalized bending rigidity $\tilde{\kappa}(q)$ exhibits a larger deviation from the harmonic approximation at scales of the order of $10^{-1} \AA^{-1}$. However, the effect is relatively small. For $q \gtrsim 10^{-2} \AA^{-1}, \tilde{\kappa}(q)$ and $\tilde{b}(q)$ differ from the corresponding functions in the harmonic approximation by less than $10 \%$. In the long-wavelength region $q \lesssim 10^{-2} \AA^{-1}$, instead, $\tilde{\kappa}(q)$ and $\tilde{b}(q)$ agree within $9 \%$ with the renormalized rigidity $\tilde{\kappa}_{1}(q)$ and elastic modulus $\tilde{b}_{1}(q)$ of a single membrane with bare bending stiffness $\bar{\kappa}_{0}$ and Young modulus $2 Y$. In particular, comparing amplitudes of the leading scaling behavior in the limit $q \rightarrow 0$ shows that $\tilde{\kappa}(q)$ and $\tilde{b}(q)$ deviate from $\tilde{\kappa}_{1}(q)$ and $\tilde{b}_{1}(q)$ by approximately $3 \%$ and $6 \%$, respectively [80]. An explicit comparison is illustrated graphically in Fig. 7.

The effects of thermal renormalizations are more pronounced at $T=1500 \mathrm{~K}$, as Fig. 8(c) shows. Within the considered model, the amplitude of the long-wavelength power-law behavior $\tilde{\kappa}(q)=T z_{1}^{\prime \prime} q^{-\eta}$ differs from the scaling limit of $\tilde{\kappa}_{1}(q), \tilde{\kappa}_{1}(q)=\bar{z}_{1} \bar{\kappa}_{0}\left(q_{2 *} / q\right)^{\eta}$, by approximately $10 \%$ [80].

In correspondence with crossover regions for $\tilde{\kappa}(q)$, the renormalized elastic coefficient $\tilde{b}(q)$ exhibits a flection (see Fig. 7). Since $b_{0}(q)$ is assumed to be wave-vector indepen- dent, this behavior reflects corresponding crossovers in the polarization function $I(q)$.

As a final remark, it should be noted that features in the reported results with $q$ of the order of $1 \AA^{-1}$ and their contribution to the renormalization of the long-wavelength behavior can be sensitive to microscopic effects not captured by the continuum approximation employed here. Renormalizations beyond the continuum model are expected to grow with increasing temperature and to become important when strong nonlinear effects occur at microscopic scales.

\section{INCLUSION OF INTERLAYER FLEXURAL NONLINEARITIES}

In the model considered in this paper, nonlinearities in $\bar{h}=h_{1}-h_{2}$ and $\bar{u}_{\alpha}$ have been neglected. As a result of the harmonic approximation, however, Eq. (23) fails to recover the theory of two independent nonlinearly fluctuating layers in the complementary limit $g_{1}, g_{2}, g_{3}, g_{4} \rightarrow 0$. A minimal extension of the theory necessary to connect this limiting regime can be constructed by including nonlinearities in the interlayer flexural field $\bar{h}$, while neglecting anharmonicity in in-plane displacement fields. With this extension, an analog of Eq. (23) reads

$$
\begin{aligned}
\tilde{H}= & \frac{1}{2} \int \mathrm{d}^{2} x\left[\kappa\left(\partial^{2} h_{1}\right)^{2}+\lambda\left(u_{1 \alpha \alpha}\right)^{2}+2 \mu\left(u_{1 \alpha \beta}\right)^{2}\right. \\
& +\kappa\left(\partial^{2} h_{2}\right)^{2}+\lambda\left(u_{2 \alpha \alpha}\right)^{2}+2 \mu\left(u_{2 \alpha \beta}\right)^{2} \\
& +\frac{g_{1}}{l^{2}} \bar{h}^{2}+\frac{g_{2}}{l^{2}}\left(\bar{u}_{\alpha}+(l+\bar{h}) \partial_{\alpha} h\right)^{2}+\frac{g_{3}}{2 l}\left(u_{1 \alpha \alpha}+u_{2 \alpha \alpha}\right) \\
& +\frac{g_{4}}{l}\left(\left(\bar{u}_{x}+(l+\bar{h}) \partial_{x} h\right)\left(u_{x x}-u_{y y}\right)\right. \\
& \left.\left.-2\left(\bar{u}_{y}+(l+\bar{h}) \partial_{y} h\right) u_{x y}\right)\right],
\end{aligned}
$$

where $u_{i \alpha \beta}=\frac{1}{2}\left(\partial_{\alpha} u_{i \beta}+\partial_{\beta} u_{i \alpha}+\partial_{\alpha} h_{i} \partial_{\beta} h_{i}\right)$ are approximate strain tensors of the $i$ th layer. For $g_{1}, g_{2}, g_{3}, g_{4}=0$, Eq. (61) reduces to two copies of the well-known nonlinear effective theory for monolayer membranes $[4,6,8,12]$.

Developing a general theory for weakly coupled membranes with large interlayer-distance fluctuations is a complex problem. If the field $\bar{h}$ is regarded as critical, with a propagator scaling as $q^{-4}$, power counting indicates an infinite number of relevant and marginal perturbations (see, e.g., Ref. [81] for a related analysis). Eq. (61), therefore, is not a general Hamiltonian but rather, a minimal extension which connects the harmonic theory to a nonlinear decoupled regime of the two membranes.

The theory defined by Eq. (4) is invariant under the transformations (see Refs. [2,8])

$$
\begin{aligned}
h_{1}(\mathbf{x}) & \rightarrow h_{1}(\mathbf{x})+A_{\alpha} x_{\alpha}+B \\
h_{2}(\mathbf{x}) & \rightarrow h_{2}(\mathbf{x})+A_{\alpha} x_{\alpha}+B \\
u_{1 \alpha}(\mathbf{x}) & \rightarrow u_{1 \alpha}(\mathbf{x})-A_{\alpha}\left(\frac{l}{2}+h_{1}(\mathbf{x})\right)-\frac{1}{2} A_{\alpha} A_{\beta} x_{\beta}+B_{\alpha}^{\prime}, \\
u_{2 \alpha}(\mathbf{x}) & \rightarrow u_{2 \alpha}(\mathbf{x})+A_{\alpha}\left(\frac{l}{2}-h_{2}(\mathbf{x})\right)-\frac{1}{2} A_{\alpha} A_{\beta} x_{\beta}+B_{\alpha}^{\prime},
\end{aligned}
$$


which represent deformed versions of rotations in the embedding space, adapted to match the neglection of in-plane nonlinearities.

Qualitatively, in the case of bilayer graphene, anharmonic terms in $\bar{h}$ are expected to play a minor role.

\section{SUMMARY AND CONCLUSIONS}

In summary, this paper analyzed the statistical mechanics of equilibrium thermal ripples in a tensionless sheet of suspended bilayer graphene. The individual graphene membranes forming the bilayer were described as continuum 2D media with finite bending rigidity and elastic moduli. For the description of interlayer interactions, a phenomenological model in the spirit of elasticity theory was constructed. Although the fluctuation energy is expanded to leading order for small deformations, anharmonicities emerge as a necessary consequence of rotational invariance, which forces the energy to be expressed in terms of nonlinear scalar strains.

For explicit calculations, the model was simplified by neglecting nonlinearities in the interlayer shear and compression modes, and by dropping anharmonic interactions of collective in-plane displacements. An effective theory describing the statistics of soft flexural fluctuations was then derived by Gaussian integration. The resulting model is controlled by bending rigidity and a long-range interactions between local Gaussian curvatures and it is identical in form to the analog theory for a monolayer membrane. However, the bare bending rigidity $\kappa_{0}(q)$ exhibits a strong wave-vector dependence at mesoscopic scales. Relevant phenomenological parameters governing the strength of interlayer interactions were derived in the case of AB-stacked bilayer graphene through $a b$ initio density-functional theory calculations, by combining an exchange-correlation functional within the Perdew-BurkeErnzerhoff approximation and van der Waals corrections in the Grimme-D2 model.

Due to the formal equivalence to a corresponding singlemembrane theory, the statistical mechanics of fluctuations can be addressed by well-developed approaches. In this paper, the field theory integral equations of motion were solved within the SCSA. To access correlation functions at arbitrary wave-vector $q$, SCSA equations were solved numerically by an iterative algorithm.

The numerical solutions recover with good accuracy analytical SCSA predictions for universal properties in the long-wavelength scaling behavior. At mesoscopic lengths, the calculated correlation functions exhibit a rich crossover behavior, driven by the harmonic coupling between bending and interlayer shear and by renormalizations due to nonlinear interactions.

In the final part of the paper, a minimal extension of the theory, including nonlinearities in the flexural fields of both layers was briefly discussed.

\section{ACKNOWLEDGMENTS}

The work of A.M. and M.I.K. was supported by the Netherlands Organisation for Scientific Research (NWO) via the Spinoza Prize. D.S. acknowledges financial support from the EU through the MSCA Project No. 796795 SOT-2DvdW. Part of this work was carried out on the Dutch national einfrastructure with the support of SURF Cooperative.

\section{APPENDIX A: DERIVATION OF THE EFFECTIVE THEORY FOR FLEXURAL FLUCTUATIONS}

The statistical distribution for fluctuations of $h(\mathbf{x})$ and $u_{\alpha}(\mathbf{x})$ is obtained from the complete Gibbs distribution of the problem by integration over $\bar{h}(\mathbf{x})$ and $\bar{u}_{\alpha}(\mathbf{x})$ :

$$
P\left[h(\mathbf{x}), u_{\alpha}(\mathbf{x})\right]=\frac{1}{Z} \int\left[\mathrm{d} \bar{h} \mathrm{~d} \bar{u}_{\alpha}\right] \mathrm{e}^{-\tilde{H} / T} .
$$

This leads to an effective Hamiltonian:

$$
\tilde{H}_{\text {eff }}^{\prime}=-T \ln \left\{\int\left[\mathrm{d} \bar{h} \mathrm{~d} \bar{u}_{\alpha}\right] \mathrm{e}^{-\tilde{H} / T}\right\} .
$$

Since $\tilde{H}$, Eq. (23), is quadratic in $\bar{u}_{\alpha}(\mathbf{x})$ and $\bar{h}(\mathbf{x})$ functional integrations over $\bar{h}(\mathbf{x}), \bar{u}_{\alpha}(\mathbf{x})$, take the form of general Gaussian integrals

$$
\begin{aligned}
z\left[J_{a}\right]= & \int\left[\mathrm{d} \varphi_{a}\right] \exp \left\{-\left[\frac{1}{2} \int_{\mathbf{x}} \int_{\mathbf{x}^{\prime}} B_{a b}\left(\mathbf{x}, \mathbf{x}^{\prime}\right) \varphi_{a}(\mathbf{x}) \varphi_{b}\left(\mathbf{x}^{\prime}\right)\right.\right. \\
& \left.\left.+\int_{\mathbf{x}} J_{a}(\mathbf{x}) \varphi_{a}(\mathbf{x})\right]\right\}
\end{aligned}
$$

where $J_{a}(\mathbf{x})$ is a space-dependent source and $B_{a b}\left(\mathbf{x}, \mathbf{x}^{\prime}\right)=$ $B_{b a}\left(\mathbf{x}^{\prime}, \mathbf{x}\right)$ is a symmetric, positive definite operator independent of $J_{a}(\mathbf{x})$. By explicit calculation, the Gaussian integral reads

$$
z\left[J_{a}(\mathbf{x})\right]=\mathcal{Z} \exp \left[\frac{1}{2} \int_{\mathbf{x}} \int_{\mathbf{x}^{\prime}} \Delta_{a b}\left(\mathbf{x}, \mathbf{x}^{\prime}\right) J_{a}(\mathbf{x}) J_{b}\left(\mathbf{x}^{\prime}\right)\right],
$$

where the propagator $\Delta_{a b}\left(\mathbf{x}, \mathbf{x}^{\prime}\right)$ is the inverse of $B_{a b}\left(\mathbf{x}, \mathbf{x}^{\prime}\right)$,

$$
\int \mathrm{d}^{2} x^{\prime \prime} B_{a c}\left(\mathbf{x}, \mathbf{x}^{\prime \prime}\right) \Delta_{c b}\left(\mathbf{x}^{\prime \prime}, \mathbf{x}^{\prime}\right)=\delta_{a b} \delta\left(\mathbf{x}-\mathbf{x}^{\prime}\right),
$$

and the normalization $\mathcal{Z}$, formally given by

$$
\mathcal{Z}=\int\left[\mathrm{d} \varphi_{a}\right] \mathrm{e}^{-\frac{1}{2} \int \mathrm{d}^{2} x \int \mathrm{d}^{2} x^{\prime} B_{a b}\left(\mathbf{x}, \mathbf{x}^{\prime}\right) \varphi_{a}(\mathbf{x}) \varphi_{b}\left(\mathbf{x}^{\prime}\right)},
$$

is independent of the source $J_{a}(\mathbf{x})$.

To integrate over $\bar{u}_{\alpha}$, it is convenient to shift variables by the replacement $\bar{u}_{\alpha} \rightarrow \bar{u}_{\alpha}-l \partial_{\alpha} h$. With these shifted variables, Eq. (23) reads, up to boundary terms,

$$
\begin{aligned}
\tilde{H}= & \int \mathrm{d}^{2} x\left[\kappa\left(\partial^{2} h\right)^{2}+\lambda\left(u_{\alpha \alpha}\right)^{2}+2 \mu u_{\alpha \beta} u_{\alpha \beta}\right. \\
& +\frac{\kappa}{4}\left(\partial^{2} \bar{h}\right)^{2}+\frac{\lambda}{4}\left(\partial_{\alpha} \bar{u}_{\alpha}\right)^{2}+\frac{\mu}{8}\left(\partial_{\alpha} \bar{u}_{\beta}+\partial_{\beta} \bar{u}_{\alpha}\right)^{2} \\
& +\frac{(\lambda+2 \mu) l^{2}}{4}\left(\partial^{2} h\right)^{2}-\frac{(\lambda+2 \mu) l}{2}\left(\partial_{\alpha} \bar{u}_{\alpha}\right) \partial^{2} h \\
& \left.+\frac{g_{1}}{2 l^{2}} \bar{h}^{2}+\frac{g_{2}}{2 l^{2}} \bar{u}_{\alpha}^{2}+\frac{g_{3}}{2 l} \bar{h} u_{\alpha \alpha}+\frac{g_{4}}{2 l} \bar{u}_{\alpha} A_{\alpha}\right],
\end{aligned}
$$

where $A_{x}=u_{x x}-u_{y y}$ and $A_{y}=-2 u_{x y}$. From the $\bar{u}_{\alpha^{-}}$ dependent terms, we read the inverse propagator

$$
\begin{aligned}
B_{\alpha \beta}\left(\mathbf{x}, \mathbf{x}^{\prime}\right)= & \frac{1}{T}\left\{-\frac{1}{2}\left[(\lambda+\mu) \partial_{\alpha} \partial_{\beta}+\mu \delta_{\alpha \beta} \partial^{2}\right]\right. \\
& \left.+\frac{g_{2}}{l^{2}} \delta_{\alpha \beta}\right\} \delta\left(\mathbf{x}-\mathbf{x}^{\prime}\right),
\end{aligned}
$$


and the source

$$
J_{\alpha}(\mathbf{x})=\frac{1}{T}\left[\frac{(\lambda+2 \mu) l}{2} \partial_{\alpha} \partial^{2} h+\frac{g_{4}}{2 l} A_{\alpha}(\mathbf{x})\right] .
$$

The propagator $\Delta_{\alpha \beta}$, inverse of $B_{\alpha \beta}$, is then

$$
\begin{aligned}
\Delta_{\alpha \beta}\left(\mathbf{x}, \mathbf{x}^{\prime}\right)= & T \int_{\mathbf{q}}\left\{\left[\frac{P_{\alpha \beta}^{L}(\mathbf{q})}{g_{2} / l^{2}+(\lambda+2 \mu) q^{2} / 2}\right.\right. \\
& \left.\left.+\frac{P_{\alpha \beta}^{T}(\mathbf{q})}{g_{2} / l^{2}+\mu q^{2} / 2}\right] \mathrm{e}^{i \mathbf{q} \cdot\left(\mathbf{x}-\mathbf{x}^{\prime}\right)}\right\} \\
= & \frac{T}{g_{2} / l^{2}} \int_{\mathbf{q}}\left\{\left[d_{L}(q) P_{\alpha \beta}^{L}(\mathbf{q})+d_{T}(q) P_{\alpha \beta}^{T}(\mathbf{q})\right]\right. \\
& \left.\times \mathrm{e}^{i \mathbf{q} \cdot\left(\mathbf{x}-\mathbf{x}^{\prime}\right)}\right\},
\end{aligned}
$$

where $P_{\alpha \beta}^{L}(\mathbf{q})=q_{\alpha} q_{\beta} / q^{2}$ and $P_{\alpha \beta}^{T}(\mathbf{q})=\delta_{\alpha \beta}-q_{\alpha} q_{\beta} / q^{2}$ are longitudinal and transverse projectors and $d_{L}(q)$ and $d_{T}(q)$ are dimensionless functions defined in Eqs. (30). Using Eq. (A4), we obtain, up to an unimportant normalization factor,

$$
\begin{aligned}
\int\left[\mathrm{d} \bar{u}_{\alpha}\right] \mathrm{e}^{-\tilde{H} / T} \\
=\exp \left\{\frac { T } { 2 g _ { 2 } / l ^ { 2 } } \int _ { \mathbf { q } } \left[\left(d_{L}(q) P_{\alpha \beta}^{L}(\mathbf{q})\right.\right.\right. \\
\left.\left.\quad+d_{T}(q) P_{\alpha \beta}^{T}(\mathbf{q})\right) J_{\alpha}(\mathbf{q}) J_{\beta}^{*}(\mathbf{q})\right] \\
\quad-\frac{1}{T} \int \mathrm{d}^{2} x\left[\kappa\left(\partial^{2} h\right)^{2}+\lambda\left(u_{\alpha \alpha}\right)^{2}+2 \mu u_{\alpha \beta} u_{\alpha \beta}\right. \\
\left.\left.+\frac{(\lambda+2 \mu) l^{2}}{4}\left(\partial^{2} h\right)^{2}+\frac{g_{1}}{2 l^{2}} \bar{h}^{2}+\frac{g_{3}}{2 l} \bar{h} u_{\alpha \alpha}\right]\right\},
\end{aligned}
$$

where $J_{\alpha}(\mathbf{q})$ is the Fourier transform of $J_{\alpha}(\mathbf{x})$,

$$
J_{\alpha}(\mathbf{q})=\frac{1}{T}\left[-i \frac{(\lambda+2 \mu) l}{2} q_{\alpha} q^{2} h(\mathbf{q})+\frac{g_{4}}{2 l} A_{\alpha}(\mathbf{q})\right],
$$

$h(\mathbf{q})$ and $A_{\alpha}(\mathbf{q})$ being the Fourier transforms of $h(\mathbf{x})$ and $A_{\alpha}(\mathbf{x})$, respectively. After introduction of $A(\mathbf{x})=\partial_{\alpha} A_{\alpha}$ and the corresponding Fourier components $A(\mathbf{q})=i q_{\alpha} A_{\alpha}(\mathbf{q})$, an explicit calculation of Eq. (A11) gives

$$
\begin{aligned}
\int\left[\mathrm{d} \bar{u}_{\alpha}\right] \mathrm{e}^{-\tilde{H} / T} \\
=\exp \left\{-\frac{1}{T}\left[\int _ { \mathbf { q } } \left(\frac{1}{2} \kappa_{0}(q)|h(\mathbf{q})|^{2}+\mu_{0}(q)\left|u_{\alpha \beta}(\mathbf{q})\right|^{2}\right.\right.\right. \\
\quad+\frac{g_{4}^{2}}{8 g_{2}} d_{T}(\mathbf{q})\left|u_{\alpha \alpha}(\mathbf{q})\right|^{2}-\frac{g_{4} l^{2}}{4 g_{2}}(\lambda+2 \mu) d_{L}(q) q^{2} h(\mathbf{q}) A^{*}(\mathbf{q}) \\
\left.\quad+\frac{g_{4}^{2} l^{2}}{16 g_{2}^{2}}(\lambda+\mu) d_{L}(q) d_{T}(q)|A(\mathbf{q})|^{2}\right) \\
\left.\left.\quad+\int \mathrm{d}^{2} x\left(\lambda\left(u_{\alpha \alpha}\right)^{2}+\frac{\kappa}{4}\left(\partial^{2} \bar{h}\right)^{2}+\frac{g_{1}}{2 l^{2}} \bar{h}^{2}+\frac{g_{3}}{2 l} \bar{h} u_{\alpha \alpha}\right)\right]\right\},
\end{aligned}
$$

where $\kappa_{0}(q)$ and $\mu_{0}(q)$ are the $q$-dependent bending rigidity and shear modulus introduced in Eq. (31). In the derivation, it is useful to take advantage of the identity

$$
A_{\alpha}(\mathbf{q}) A_{\alpha}^{*}(\mathbf{q})=2\left|u_{\alpha \beta}(\mathbf{q})\right|^{2}-\left|u_{\alpha \alpha}(\mathbf{q})\right|^{2}
$$

As a next step, we can integrate out the $\bar{h}$ field. This generates an effective interaction between the sources $g_{3} u_{\alpha \alpha}(\mathbf{x}) /(2 T l)$, mediated by the propagator

$$
\Delta\left(\mathbf{x}, \mathbf{x}^{\prime}\right)=T \int_{\mathbf{q}} \frac{\mathrm{e}^{i \mathbf{q} \cdot\left(\mathbf{x}-\mathbf{x}^{\prime}\right)}}{g_{1} / l^{2}+\kappa q^{4} / 2}=\frac{T}{g_{1} / l^{2}} \int_{\mathbf{q}} \bar{d}(q) \mathrm{e}^{i \mathbf{q} \cdot\left(\mathbf{x}-\mathbf{x}^{\prime}\right)},
$$

the inverse of

$$
B\left(\mathbf{x}, \mathbf{x}^{\prime}\right)=\frac{1}{T}\left[\frac{\kappa}{2} \partial^{4}+\frac{g_{1}}{l^{2}}\right] \delta\left(\mathbf{x}-\mathbf{x}^{\prime}\right) .
$$

Using Eq. (A4), we then obtain

$$
\begin{aligned}
\int\left[\mathrm{d} \bar{u}_{\alpha} \mathrm{d} \bar{h}\right] \mathrm{e}^{-\tilde{H} / T} \\
=\exp \left\{-\frac{1}{T}\left[\int _ { \mathbf { q } } \left(\frac{1}{2} \kappa_{0}(q)|h(\mathbf{q})|^{2}\right.\right.\right. \\
\quad+\mu_{0}(q)\left|u_{\alpha \beta}(\mathbf{q})\right|^{2}+\lambda\left|u_{\alpha \alpha}(\mathbf{q})\right|^{2} \\
\quad+\frac{g_{4}^{2}}{8 g_{2}} d_{T}(\mathbf{q})\left|u_{\alpha \alpha}(\mathbf{q})\right|^{2}-\frac{g_{3}^{2}}{8 g_{1}} \bar{d}(q)\left|u_{\alpha \alpha}(\mathbf{q})\right|^{2} \\
\quad-\frac{g_{4} l^{2}}{4 g_{2}}(\lambda+2 \mu) d_{L}(q) q^{2} h(\mathbf{q}) A^{*}(\mathbf{q}) \\
\left.\left.\left.\quad+\frac{g_{4}^{2} l^{2}}{16 g_{2}^{2}}(\lambda+\mu) d_{L}(q) d_{T}(q)|A(\mathbf{q})|^{2}\right)\right]\right\},
\end{aligned}
$$

from which we recognize the effective Hamiltonian $\tilde{H}_{\text {eff }}^{\prime}\left[h(\mathbf{x}), u_{\alpha}(\mathbf{x})\right]$, Eq. (28) in the main text.

We finally wish to eliminate the in-plane displacement fields $u_{\alpha}(\mathbf{x})$. Neglecting, as in the main text, the interactions $\int_{\mathbf{q}}|A(\mathbf{q})|^{2}$ and $\int_{\mathbf{q}} q^{2} h(\mathbf{q}) A^{*}(\mathbf{q})$, we are led to the calculation of

$$
\tilde{H}_{\text {eff }}[h(\mathbf{x})]=-T \ln \left\{\int\left[\mathrm{d} u_{\alpha}\right] \exp \left[-\tilde{H}_{\text {eff }}^{\prime \prime} / T\right]\right\},
$$

with

$$
\begin{aligned}
\tilde{H}_{\mathrm{eff}}^{\prime \prime}= & \frac{1}{2} \int_{\mathbf{q}}\left[\kappa_{0}(q) q^{4}|h(\mathbf{q})|^{2}+\lambda_{0}(q)\left|u_{\alpha \alpha}(\mathbf{q})\right|^{2}\right. \\
& \left.+2 \mu_{0}(q)\left|u_{\alpha \beta}(\mathbf{q})\right|^{2}\right] .
\end{aligned}
$$

Although, eventually, we will assume $q$-independent Lamé coefficients $\lambda_{0}(q)$ and $\mu_{0}(q)$, it is not difficult to keep general $q$-dependent couplings in the course of the derivation.

Equation (A19) is identical in form with the standard configuration energy of a crystalline membrane, but with elastic and bending parameters replaced by the $q$-dependent functions defined in Eq. (31). Integration over $u_{\alpha}$ then proceeds in an usual way (see Chap. 6 of Ref. [1] and Refs. [7,9,10,15]).

As a first step, it is important to separate the $\mathbf{q}=0$ component of the strain tensor $u_{\alpha \beta}(\mathbf{x})$ [1] (see also Ref. [15] for an analysis of zero modes in presence of external tension):

$$
\begin{aligned}
u_{\alpha \beta}(\mathbf{x})= & u_{\alpha \beta}^{0}+c_{\alpha \beta}^{0}+\frac{1}{2} \int_{\mathbf{q}}^{\prime}\left(i q_{\alpha} u_{\beta}(\mathbf{q})+i q_{\beta} u_{\alpha}(\mathbf{q})\right. \\
& \left.+c_{\alpha \beta}(\mathbf{q})\right) \mathrm{e}^{i \mathbf{q} \cdot \mathbf{x}} .
\end{aligned}
$$


Here

$$
c_{\alpha \beta}(\mathbf{q})=\int \mathrm{d}^{2} x \mathrm{e}^{-i \mathbf{q} \cdot \mathbf{x}} \partial_{\alpha} h(\mathbf{x}) \partial_{\beta} h(\mathbf{x})
$$

is the Fourier transform of the field $c_{\alpha \beta}=\partial_{\alpha} h \partial_{\beta} h, c_{\alpha \beta}^{0}$ is its $\mathbf{q}=0$ component, and $u_{\alpha \beta}^{0}$ is the uniform component of $\left(\partial_{\alpha} u_{\beta}+\partial_{\beta} u_{\alpha}\right) / 2$. The primed integral, $\int_{\mathbf{q}}^{\prime}$, is intended to run over all nonzero wave vectors, with the $\mathbf{q}=0$ term excluded.

In the functional integral, we can consider separate integrations over uniform and finite-wavelength components. After the translation of variables $u_{\alpha \beta}^{0} \rightarrow u_{\alpha \beta}^{0}-c_{\alpha \beta}^{0}$, the integral over uniform components factorizes and gives an irrelevant normalization constant, independent on the $h(\mathbf{x})$ field.

To perform the remaining integral over the $\mathbf{q} \neq 0$ components of $u_{\alpha}$, it is convenient to decompose $c_{\alpha \beta}(\mathbf{q})$ in the form [1]

$$
c_{\alpha \beta}(\mathbf{q})=i q_{\alpha} \phi_{\beta}(\mathbf{q})+i q_{\beta} \phi_{\alpha}(\mathbf{q})+P_{\alpha \beta}^{T}(\mathbf{q}) \omega(\mathbf{q}),
$$

where $\phi_{\alpha}(\mathbf{q})$ is a two-component vector and

$$
\omega(\mathbf{q})=P_{\alpha \beta}^{T}(\mathbf{q}) c_{\alpha \beta}(\mathbf{q}) .
$$

This decomposition is possible for any 2D symmetric matrix. After the shift of integration variables $u_{\alpha}(\mathbf{x}) \rightarrow u_{\alpha}(\mathbf{x})-$ $\phi_{\alpha}(\mathbf{x})$, the Fourier components of the strain tensor become independent of $\phi_{\alpha}(\mathbf{q})$. An explicit calculation then leads to the effective Hamiltonian

$$
H_{\mathrm{eff}}=\frac{1}{2} \int_{\mathbf{q}} \kappa_{0}(q) q^{4}|h(\mathbf{q})|^{2}+\int_{\mathbf{q}}^{\prime} \frac{Y_{0}(q)}{8}|\omega(\mathbf{q})|^{2},
$$

with

$$
Y_{0}(q)=\frac{4 \mu_{0}(q)\left(\lambda_{0}(q)+\mu_{0}(q)\right)}{\lambda_{0}(q)+2 \mu_{0}(q)} .
$$

Inspecting Eq. (A23), we recognize that $\omega(\mathbf{q})=2 K(\mathbf{q}) / q^{2}$, where $K(\mathbf{q})$ is the Fourier transform of the approximate Gaussian curvature, Eq. (13). With the approximation $\lambda_{0}(q) \simeq 2 \lambda$, $\mu_{0}(q) \simeq 2 \mu, Y_{0}(q)=4 \mu_{0}\left(\lambda_{0}+\mu_{0}\right) /\left(\lambda_{0}+2 \mu_{0}\right)$, we thus obtain Eq. (34) of the main text.
[1] Statistical Mechanics of Membranes and Surfaces, 2nd ed., edited by D. R. Nelson, T. Piran, and S. Weinberg. (World Scientific, Singapore, 2004).

[2] M. J. Bowick and A. Travesset, The statistical mechanics of membranes, Phys. Rep. 344, 255 (2001).

[3] M. I. Katsnelson, The Physics of Graphene, 2nd ed. (Cambridge University Press, Cambridge, 2020).

[4] D. R. Nelson and L. Peliti, Fluctuations in membranes with crystalline and hexatic order, J. Physique 48, 1085 (1987).

[5] F. David and E. Guitter, Crumpling transition in elastic membranes: Renormalization group treatment, EPL 5, 709 (1988).

[6] J. A. Aronovitz and T. C. Lubensky, Fluctuations of Solid Membranes, Phys. Rev. Lett. 60, 2634 (1988).

[7] J. Aronovitz, L. Golubovic, and T. C. Lubensky, Fluctuations and lower critical dimensions of crystalline membranes, J. Physique 50, 609 (1989).

[8] E. Guitter, F. David, S. Leibler, and L. Peliti, Thermodynamical behavior of polymerized membranes, J. Phys. 50, 1787 (1989).

[9] P. Le Doussal and L. Radzihovsky, Self-Consistent Theory of Polymerized Membranes, Phys. Rev. Lett. 69, 1209 (1992).

[10] P. Le Doussal and L. Radzihovsky, Anomalous elasticity, fluctuations and disorder in elastic membranes, Ann. Phys. 392, 340 (2018).

[11] J.-P. Kownacki and D. Mouhanna, Crumpling transition and flat phase of polymerized phantom membranes, Phys. Rev. E 79, 040101(R) (2009).

[12] I. V. Gornyi, V. Y. Kachorovskii, and A. D. Mirlin, Rippling and crumpling in disordered free-standing graphene, Phys. Rev. B 92, 155428 (2015).

[13] A. Košmrlj and D. R. Nelson, Response of thermalized ribbons to pulling and bending, Phys. Rev. B 93, 125431 (2016).

[14] O. Coquand, K. Essafi, J.-P. Kownacki, and D. Mouhanna, Glassy phase in quenched disordered crystalline membranes, Phys. Rev. E 97, 030102(R) (2018).
[15] I. S. Burmistrov, V. Y. Kachorovskii, I. V. Gornyi, and A. D. Mirlin, Differential Poisson's ratio of a crystalline two-dimensional membrane, Ann. Phys. 396, 119 (2018).

[16] D. R. Saykin, V. Y. Kachorovskii, and I. S. Burmistrov, Disorder-induced rippled phases and multicriticality in freestanding graphene, arXiv:2003.03421.

[17] A. Mauri and M. I. Katsnelson, Scaling behavior of crystalline membranes: An $\epsilon$-expansion approach, Nucl. Phys. B 956, 115040 (2020).

[18] O. Coquand, D. Mouhanna, and S. Teber, Flat phase of polymerized membranes at two-loop order, Phys. Rev. E 101, 062104 (2020).

[19] M. I. Katsnelson and A. Fasolino, Graphene as a prototype crystalline membrane, Acc. Chem. Res. 46, 97 (2013).

[20] F. S. Schmidt, K. Svoboda, N. Lei, I. B. Petsche, L. E. Berman, C. R. Safinya, and G. S. Grest, Existence of a flat phase in red cell membrane skeletons, Science 259, 952 (1993).

[21] A. Fasolino, J. H. Los, and M. I. Katsnelson, Intrinsic ripples in graphene, Nat. Mater. 6, 858 (2007).

[22] J. H. Los, M. I. Katsnelson, O. V. Yazyev, K. V. Zakharchenko, and A. Fasolino, Scaling properties of flexible membranes from atomistic simulations: Application to graphene, Phys. Rev. B 80, 121405(R) (2009).

[23] J. C. Meyer, A. K. Geim, M. I. Katsnelson, K. S. Novoselov, D. Obergfell, S. Roth, C. Girit, and A. Zettl, On the roughness of single- and bi-layer graphene membranes, Solid State Commun. 143, 101 (2007).

[24] M. K. Blees, A. W. Barnard, P. A. Rose, S. P. Roberts, K. L. McGill, P. Y. Huang, A. R. Ruyack, J. W. Kevek, B. Kobrin, D. A. Muller, and P. L. McEuen, Graphene kirigami, Nature (London) 524, 204 (2015).

[25] R. J. T. Nicholl, H. J. Conley, N. V. Lavrik, I. Vlassiouk, Y. S. Puzyrev, V. P. Sreenivas, S. T. Pantelides, and K. I. Bolotin, The 
effect of intrinsic crumpling on the mechanics of free-standing graphene, Nat. Commun. 6, 8789 (2015).

[26] R. J. T. Nicholl, N. V. Lavrik, I. Vlassiouk, B. R. Srijanto, and K. I. Bolotin, Hidden Area and Mechanical Nonlinearities in Freestanding Graphene, Phys. Rev. Lett. 118, 266101 (2017).

[27] G. López-Polín, M. Jaafar, F. Guinea, R. Roldán, C. GómezNavarro, and J. Gómez-Herrero, The influence of strain on the elastic constants of graphene, Carbon 124, 42 (2017).

[28] C. S. Ruiz-Vargas, H. L. Zhuang, P. Y. Huang, A. M. van der Zande, S. Garg, P. L. McEuen, D. A. Muller, R. G. Hennig, and J. Park, Softened elastic response and unzipping in chemical vapor deposition graphene membranes, Nano Lett. 11, 2259 (2011).

[29] M. Pozzo, D. Alfè, P. Lacovig, P. Hofmann, S. Lizzit, and A. Baraldi, Thermal Expansion of Supported and Freestanding Graphene: Lattice Constants Versus Interatomic Distance, Phys. Rev. Lett. 106, 135501 (2011).

[30] J. K. Schoelz, P. Xu, V. Meunier, P. Kumar, M. Neek-Amal, P. M. Thibado, and F. M. Peeters, Graphene ripples as a realization of a two-dimensional Ising model: A scanning tunneling microscope study, Phys. Rev. B 91, 045413 (2015).

[31] A. Georgi, P. Nemes-Incze, B. Szafranek, D. Neumaier, V. Geringer, M. Liebmann, and M. Morgenstern, Apparent rippling with honeycomb symmetry and tunable periodicity observed by scanning tunneling microscopy on suspended graphene, Phys. Rev. B 94, 184302 (2016).

[32] F. Colangelo, P. Pingue, V. Mišeikis, C. Coletti, F. Beltram, and S. Roddaro, Mapping the mechanical properties of a graphene drum at the nanoscale, 2D Mater. 6, 025005 (2019).

[33] J. H. Los, J. M. H. Kroes, K. Albe, R. M. Gordillo, M. I. Katsnelson, and A. Fasolino, Extended Tersoff potential for boron nitride: Energetics and elastic properties of pristine and defective $h$-BN, Phys. Rev. B 96, 184108 (2017).

[34] K. V. Zakharchenko, J. H. Los, M. I. Katsnelson, and A. Fasolino, Atomistic simulations of structural and thermodynamic properties of bilayer graphene, Phys. Rev. B 81, 235439 (2010)

[35] J. Hašík, E. Tosatti, and R. Martoňák, Quantum and classical ripples in graphene, Phys. Rev. B 97, 140301(R) (2018).

[36] C. P. Herrero and R. Ramírez, Thermodynamic properties of graphene bilayers, Phys. Rev. B 101, 035405 (2020).

[37] C. Androulidakis, K. Zhang, M. Robertson, and S. Tawfick, Tailoring the mechanical properties of 2D materials and heterostructures, 2D Mater. 5, 032005 (2018).

[38] J. H. Kim, J. H. Jeong, N. Kim, R. Joshi, and G.-H. Lee, Mechanical properties of two-dimensional materials and their applications, J. Phys. D: Appl. Phys. 52, 083001 (2018).

[39] P. L. de Andres, F. Guinea, and M. I. Katsnelson, Bending modes, anharmonic effects, and thermal expansion coefficient in single-layer and multilayer graphene, Phys. Rev. B 86, 144103 (2012).

[40] G. Wang, Z. Dai, J. Xiao, S. Z. Feng, C. Weng, L. Liu, Z. Xu, R. Huang, and Z. Zhang, Bending of Multilayer Van Der Waals Materials, Phys. Rev. Lett. 123, 116101 (2019).

[41] F. Pan, G. Wang, L. Liu, Y. Chen, Z. Zhang, and X. Shi, Bending induced interlayer shearing, rippling and kink buckling of multilayered graphene sheets, J. Mech. Phys. Solids 122, 340 (2019).

[42] E. Han, J. Yu, E. Annevelink, J. Son, D. A. Kang, K. Watanabe, T. Taniguchi, E. Ertekin, P. Y. Huang, and A. M. van der Zande,
Ultrasoft slip-mediated bending in few-layer graphene, Nat. Mater. 19, 305 (2020).

[43] D.-B. Zhang, E. Akatyeva, and T. Dumitrică, Bending Ultrathin Graphene at the Margins of Continuum Mechanics, Phys. Rev. Lett. 106, 255503 (2011).

[44] L. D. Landau and E. M. Lifshitz, Theory of Elasticity (Pergamon Press, Oxford, 1970).

[45] N. Lindahl, D. Midtvedt, J. Svensson, O. A. Nerushev, N. Lindvall, A. Isaacsson, and E. E. B. Campbell, Determination of the bending rigidity of graphene via electrostatic actuation of buckled membranes, Nano Lett. 12, 3526 (2012).

[46] X. Chen, C. Yi, and C. Ke, Bending stiffness and interlayer shear modulus of few-layer graphene, Appl. Phys. Lett. 106, 101907 (2015).

[47] J. Toner, New Phase of Matter in Lamellar Phases of Tethered, Crystalline Membranes, Phys. Rev. Lett. 64, 1741 (1990).

[48] E. Guitter, Anharmonic theory of a stack of tethered membranes, J. Phys. 51, 2407 (1990).

[49] Y. Hatwalne and S. Ramaswamy, Scale-dependence of elastic constants in the decoupled lamellar phase of tethered crystalline membranes, arXiv:cond-mat/0009294.

[50] A. K. Singh and R. G. Hennig, Scaling relation for thermal ripples in single and multilayer graphene, Phys. Rev. B 87, 094112 (2013).

[51] D. Gazit, Structure of physical crystalline membranes within the self-consistent screening approximation, Phys. Rev. E 80, 041117 (2009).

[52] As discussed in Ref. [12], the comparison between curvature and elastic energies becomes nontrivial if the problem is analyzed in a large $d$-limit at fixed internal dimension $D=2$.

[53] M. Zelisko, F. Ahmadpoor, H. J. Gao, and P. Sharma, Determining the Gaussian Modulus and Edge Properties of 2D Materials: From Graphene to Lipid Bilayers, Phys. Rev. Lett. 119, 068002 (2017).

[54] M. I. Katsnelson and A. Fasolino, Solvent-driven formation of bolaamphiphilic vesicles, J. Phys. Chem. B 110, 30 (2006).

[55] O. V. Manyuhina, J. J. Hetzel, M. I. Katsnelson, and A. Fasolino, Non-spherical shapes of capsules within a fourthorder curvature model, Eur. Phys. J. E 32, 223 (2010).

[56] H. Kleinert, Membrane stiffness from van der Waals forces, Phys. Lett. A 136, 253 (1989).

[57] A. Mauri and M. I. Katsnelson, Thermal fluctuations in crystalline membranes with long-range dipole-dipole interactions, Ann. Phys. 412, 168016 (2020).

[58] F. Guinea, P. Le Doussal, and K. J. Wiese, Collective excitations in a large- $d$ model for graphene, Phys. Rev. B 89, 125428 (2014).

[59] D. Gazit, Correlation between charge inhomogeneities and structure in graphene and other electronic crystalline membranes, Phys. Rev. B 80, 161406(R) (2009).

[60] As in Sec. II A, the contributions of in-plane modes $\left(\partial^{2} u_{\alpha}\right)^{2}$ to $\left(\partial^{2} \mathbf{r}\right)^{2}$ will eventually be neglected. The chosen curvature energy is thus equivalent, to leading order, to alternative expressions such as Eq. (6).

[61] As discussed above, local interactions do not exhaust all possibilities due to the presence of infinite-range van der Waals interactions [56] and coupling with gapless electrons. Effects of nonlocal interactions are beyond the scope of this paper, and will be neglected. 
[62] It can be shown that the most general rotationally invariant tensor is a linear combination of products of identity tensors $\delta_{i j}$ and at most one fully antisymmetric tensor $\epsilon_{i j k}$. For a proof, see $\mathrm{H}$. Jeffreys, On isotropic tensors, Math. Proc. Camb. Philos. Soc. 73, 173 (1973). In Eq. (17), pseudoscalar functions constructed via vector products are ruled out by inversion symmetry and only scalar products need to be considered.

[63] L. M. Malard, M. H. D. Guimarães, D. L. Mafra, M. S. C. Mazzoni, and A. Jorio, Group-theory analysis of electrons and phonons in $N$-layer graphene systems, Phys. Rev. B 79, 125426 (2009).

[64] In the case of ABA-stacked graphite, the symmetry group includes symmetry for $z \rightarrow-z$ and $c_{16}=0$.

[65] I. S. Burmistrov, I. V. Gornyi, V. Y. Kachorovskii, M. I. Katsnelson, and A. D. Mirlin, Quantum elasticity of graphene: Thermal expansion coefficient and specific heat, Phys. Rev. B 94, 195430 (2016).

[66] G. Savini, Y. J. Dappe, S. Öberg, J. C. Charlier, M. I. Katsnelson, and A. Fasolino, Bending modes, elastic constants and mechanical stability of graphitic systems, Carbon 49, 62 (2011).

[67] A. Bosak, M. Krisch, M. Mohr, J. Maultzsch, and C. Thomsen, Elasticity of single-crystalline graphite: Inelastic x-ray scattering study, Phys. Rev. B 75, 153408 (2007).

[68] We note, however, that in Ref. [48] a perturbation analog to a finite $g_{3}$ was identified as potentially important within the framework of three-dimensional continuum theories of stacks of membranes.

[69] E. Cadelano, S. Giordano, and L. Colombo, Interplay between bending and stretching in carbon nanoribbons, Phys. Rev. B 81, 144105 (2010).

[70] L. J. Karssemeijer and A. Fasolino, Phonons of graphene and graphitic materials derived from the empirical potential LCBOPII, Surf. Sci. 605, 1611 (2011).

[71] K. H. Michel, S. Costamagna, and F. M. Peeters, Theory of anharmonic phonons in two-dimensional crystals, Phys. Rev. B 91, 134302 (2015).
[72] X. Wei, B. Fragneaud, C. A. Marianetti, and J. W. Kysar, Nonlinear elastic behavior of graphene: $A b$ initio calculations to continuum descriptions, Phys. Rev. B 80, 205407 (2009).

[73] P. Giannozzi, S. Baroni, N. Bonini, M. Calandra, R. Car, C. Cavazzoni, D. Ceresoli, G. L. Chiarotti, M. Cococcioni, and I. Dabo, Quantum Espresso: A modular and open-source software project for quantum simulations of materials, J. Phys.: Cond. Mat. 21, 395502 (2009).

[74] J. P. Perdew, K. Burke, and M. Ernzerhof, Generalized Gradient Approximation Made Simple, Phys. Rev. Lett. 77, 3865 (1996).

[75] S. Grimme, Semiempirical GGA-type density functional constructed with a long-range dispersion correction, J. Comput. Chem. 27, 1787 (2006).

[76] D. R. Saykin, I. V. Gornyi, V. Y. Kachorovskii, and I. S. Burmistrov, Absolute Poisson's ratio and the bending rigidity exponent of a crystalline two-dimensional membrane, Ann. Phys. 414, 168108 (2020).

[77] See Supplemental Material at https://link.aps.org/supplemental/ 10.1103/PhysRevB.102.165421 for data sets used in the calculations.

[78] Numerical interpolations were performed by the functions SCIPY.INTERPOLATE.PCHIPINTERPOLATOR, which implements a piecewise-cubic Hermite interpolating polynomial algorithm. For numerical integration, we used the adaptive integration method implemented in SCIPY.INTEGRATE.QUAD. Both functions are distributed in the Scipy library (version 0.19.1).

[79] M. I. Katsnelson, Flexuron: A self-trapped state of electron in crystalline membranes, Phys. Rev. B 82, 205433 (2010).

[80] As discussed in Sec. IV, both for monolayer and bilayer graphene, the ratio $z_{1}^{2} / z_{2}$ between amplitudes controlling the power-law behaviors $G^{-1}(q)=z_{1} q^{4-\eta}$ and $\tilde{b}(q)=z_{2} q^{\eta_{u}}$ is consistent with the universal value $z_{1}^{2} / z_{2}=\frac{3}{16 \pi} \frac{\Gamma^{2}(1+\eta / 2) \Gamma(1-\eta)}{\Gamma^{2}(2-\eta / 2) \Gamma(2+\eta)} \simeq$ $0.17813212 \ldots$.

[81] K. J. Wiese, Classification of perturbations for membranes with bending rigidity, Phys. Lett. B 387, 57 (1996). 